\title{
Using transcranial direct-current stimulation (tDCS) to understand cognitive processing
}

\author{
Robert M. G. Reinhart ${ }^{1}$ • Josh D. Cosman ${ }^{2}$ • \\ Keisuke Fukuda ${ }^{3} \cdot$ Geoffrey F. Woodman ${ }^{4}$
}

Published online: 1 November 2016

(C) The Psychonomic Society, Inc. 2016

\begin{abstract}
Noninvasive brain stimulation methods are becoming increasingly common tools in the kit of the cognitive scientist. In particular, transcranial direct-current stimulation (tDCS) is showing great promise as a tool to causally manipulate the brain and understand how information is processed. The popularity of this method of brain stimulation is based on the fact that it is safe, inexpensive, its effects are long lasting, and you can increase the likelihood that neurons will fire near one electrode and decrease the likelihood that neurons will fire near another. However, this method of manipulating the brain to draw causal inferences is not without complication. Because tDCS methods continue to be refined and are not yet standardized, there are reports in the literature that show some striking inconsistencies. Primary among the complications of the technique is that the tDCS method uses two or more electrodes to pass current and all of these
\end{abstract}

Robert M. G. Reinhart

rmgr@bu.edu

$\triangle$ Geoffrey F. Woodman

geoffrey.f.woodman@vanderbilt.edu

1 Department of Psychological and Brain Sciences, Center for Research in Sensory Communications and Neural Technology, Center for Systems Neuroscience, Boston University, Boston, MA 02215, USA

2 Department of Translational Medicine, Pfizer Inc., Cambridge, MA 02215, USA

3 Department of Psychology, University of Toronto Mississauga, Mississauga, ON L5L 1C6, Canada

4 Department of Psychology, Center for Integrative and Cognitive Neuroscience, Vanderbilt Vision Research Center, Vanderbilt University, Nashville, TN 37240, USA electrodes will have effects on the tissue underneath them. In this tutorial, we will share what we have learned about using tDCS to manipulate how the brain perceives, attends, remembers, and responds to information from our environment. Our goal is to provide a starting point for new users of tDCS and spur discussion of the standardization of methods to enhance replicability.

Keywords Cognitive neuroscience $\cdot$ Methods: Direct brain stimulation

\section{Introduction}

As cognitive scientists, we usually manipulate aspects of the tasks we have observers perform and measure how their behavior or brain activity changes. The inferences drawn from these manipulations are the bedrock of all cognitive science and have shaped theories of perception, attention, and information processing more generally. However, it is also possible to see what happens when the brain itself is changed. That is, we can directly manipulate the brain and see how that changes information processing when the task remains the same. Then, we can infer that the aspect we changed is necessary to perform the computations required in our task of interest.

The transcranial Direction-Current Stimulation (tDCS) technique has become an increasingly popular way to manipulate brain activity, but this popularity has come with growing pains. For example, recent selective metaanalyses suggest that the tDCS literature is filled with inconsistencies that make definitive conclusions hard to draw (Horvath et al., 2015a,b; but see Antal et al., 2015; Price \& Hamilton, 2015). As we will discuss, there are a number of ways in which well-intentioned cognitive 
scientists can fail to obtain interpretable results using tDCS. Thus, one goal in this tutorial is to contribute to work already underway to standardize methods for using tDCS to study cognitive processing in the brain. A number of groups are leading this effort and the reader would be well served to read the excellent papers available on tDCS methods (DaSilva et al., 2011; Filmer et al., 2014; Fregni et al., 2006a,b; Kuo \& Nitsche, 2012; Santarnecchi et al., 2015; Utz et al., 2010). Our novel contribution here is to make concrete the logic behind several of our key methodological choices, specifically the choice of electrode configurations, our methods for modeling of current flow which can be time consuming for a new user to accumulate across many sources, and the biophysical background that justifies these choices.

Our second goal is to explain to the reader the major pitfalls that can contribute to inconsistent results across experiments and laboratories, as we see them. We expect that our reader is new to the tDCS method. We also expect that our audience is an avid reader of Attention, Perception \& Psychophysics, but new to the biophysics of electrical fields in the brain. The format of this type of article provides more space to cover the basic background and describe how to perform the experiments than some of the other types of useful review papers that we also recommend (e.g., DaSilva et al., 2011; Filmer et al., 2014; Santarnecchi et al., 2015; Utz et al., 2010).

Beginning to use a new method for studying the brain is always nerve racking. This is particularly the case when your method involves changing how the brain works. To help the new users, we begin by discussing the nature of the stimulation and what we know about the biophysical basis of the effects in the brain. We then turn to the practical matters of choosing electrode configurations, modeling current flow, and combining tDCS with other neuroscientific techniques. We selected these topics because they are most essential for someone who would like to be an informed consumer of tDCS research, or someone looking for a springboard to begin their own tDCS research.

\section{What is tDCS?}

Using tDCS involves passing current through the skin, skull, and brain with a direct-current device. A battery is a device that we all have experience with that delivers direct current. If you connect a fresh 9-volt battery to an oscilloscope, you will see the scope jump from 0 volts to 9 volts as soon as the battery terminals (the plus and the minus) are attached to the two probes (see Fig. 1A). If you take one of these probes off, then the measured voltage returns to 0 volts as the circuit is broken. Using tDCS
A

Direct current

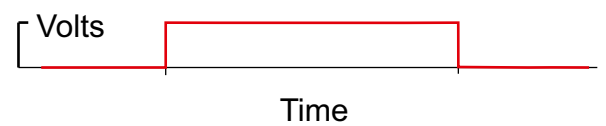

B

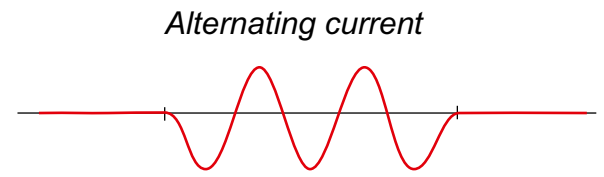

Fig. 1 Illustration of the nature of direct current versus alternating current. (A) Direct current involves passing a constant current through a circuit. (B) Alternating current oscillates between negative and positive voltage. A third type of transcranial stimulation is noise stimulation, in which the voltage steps randomly across time

to manipulate activity in the brain involves passing precisely this same kind of constant current through the tissue at a very low intensity, typically for a fairly long period of time (e.g., $20 \mathrm{~min}$ ).

Direct current differs from alternating current that changes continuously across time (see Fig. 1B). We all have experience with alternating current too. Alternating current comes out of our wall outlets. In the United States this current alternates at $60 \mathrm{~Hz}$ (e.g., it goes from 0 to -120 volts, past 0 again, to +120 volts, 60 times $/ \mathrm{s})$. Here we will focus on tDCS and not discuss the use of transcranial Alternating-Current Stimulation (tACS) at length. We chose to keep our focus on tDCS because there are important differences between these types of current. The key difference for present purposes is that the skull works like a low-pass filter in the frequency domain due to its impedance characteristics relative to the surrounding tissue (Nunez \& Srinivasan, 2006). This filtering characteristic means that the skull effectively attenuates more and more of the tACS applied as higher frequencies are used, filtering much of it above $25 \mathrm{~Hz}$. This makes it difficult to lump tACS and tDCS studies together and draw general conclusions about efficacy. Another variant of stimulation that lies in the conceptual space between tACS and tDCS is transcranial Random Noise Stimulation (or tRNS). This amounts to stimulation that is carried by a direct-current shift with the absolute current changing randomly and rapidly across time on top of the direct-current shift. The use of tACS and tRNS can have advantages, such as reduced cutaneous perception of stimulation by subjects (Ambrus et al., 2011; Paulus, 2011), but here we will focus on tDCS because it is the most common and well-understood type of transcranial electrical stimulation, and we refer the interested reader to the developing literature on tACS and tRNS (Antal \& Herrmann, 2016; Reato et al., 2013).

The tDCS technique essentially involves hooking up the positive terminal on a battery (known as the anode) 


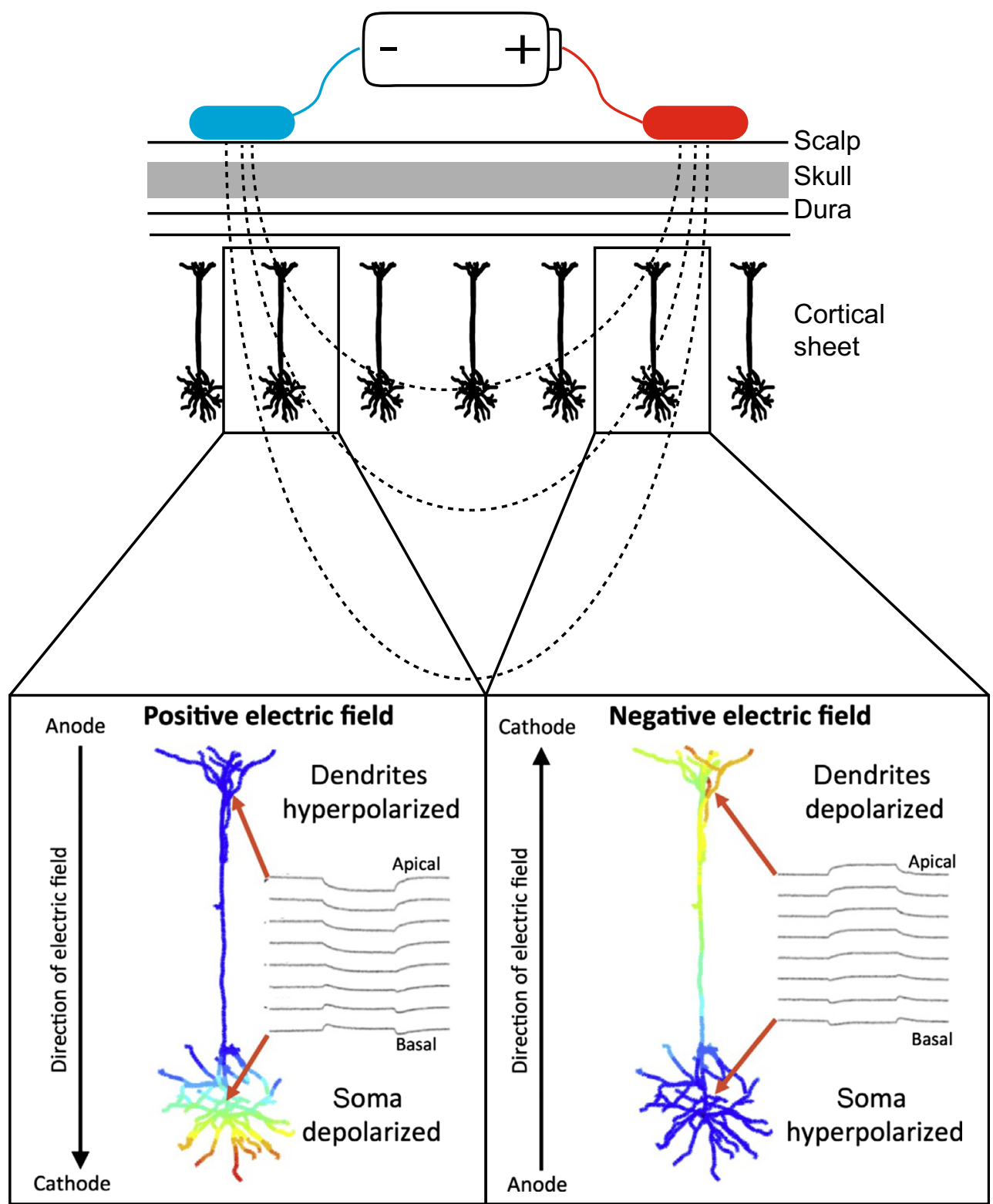

Fig. 2 Illustration of the influence of the bipolar electrical field on neurons close to the anode (blue) versus cathode (red). The top panel shows a schematic representation of the electrodes on the scalp and the electrical field that is generated. This depolarizes the soma, or cell body of neurons near the anodal electrode bringing the closer to their thresholds

to one place on the head and the negative terminal (known as the cathode) to another place on the head. Figure 2 shows a simplified schematic of such a circuit.

Typically, the electrical connection between the battery terminals is made through wires, conductive rubber pads, and then saline-soaked sponges. The tDCS devices that you can purchase from various vendors simply place some circuitry and software between the battery and the electrodes on the head. This circuitry performs several simple functions. It allows the user to turn up or down the intensity of current being delivered, it is designed to have that current ramp up or down slowly across time, for firing an action potential. The ionic gradients near the cathode have the opposite effect. This is why the logical placement of both electrodes is crucial in tDCS experiments. Bottom panels are adapted with permission from Rahman et al. (2013) and the Journal of Physiology

and can maintain constant current flow across changes in resistance. The advantage of having this software to ramp the current up and down slowly is that sudden changes in voltage can harm tissue and invoke painful sensations. Most of these devices also pass very small pulses riding on top of the direct current to test the quality of the circuit through the head as stimulation is delivered. This is how they can signal to the user that the resistance has become too high in the circuit. Resistance is simply how much the material in a circuit reduces the flow of electrical current through it, so that when resistance is high less current will flow. 
It is important to keep in mind that electrical current is like water. It follows the path of least resistance. The lowest resistance tissue that the current encounters on its journey through the head is the skin and the brain. However, between these two fairly low resistance types of tissue is the relatively high-resistance skull. This means that a significant amount of the current we try to pass through the brain with tDCS is actually shunted through the scalp and does not penetrate the skull. Based on classic work with cadaver human and monkey skulls, we know that approximately $45 \%$ of the current that tDCS electrodes deliver to the scalp makes it through the skull to the brain (Burger \& van Milaan, 1943; Rush \& Driscoll, 1968).

What happens in the brain tissue after you have begun to deliver current is a matter of active research, biophysical modeling, and debate. Next we will present the current working hypothesis.

\section{Biophysical basis}

Here is what is believed to unfold as the bipolar electric field is maintained on a subject's head. The potential difference between the two electrode contacts on the head creates an electromotive force that pushes positively charged ions (like $\mathrm{K}^{+}, \mathrm{Na}^{+}, \mathrm{Ca}^{2+}$ ) away from the anode and pulls those positive ions toward the cathode. The opposite happens with negatively charged ions. So anions (like $\mathrm{Cl}^{-}$) are pulled toward the anode and pushed away from the cathode. Although these ionic gradient have effects on cells that are immediately below the electrodes and oriented perpendicular to the stimulating electrodes, as shown in Fig. 2, there is also evidence from slice work suggesting that the strongest effects may actually be due to the radial electrical fields generated along the cortical sheet, extending parallel to the skull (Rahman et al., 2013). This means that ionic gradients are established to the left and right of your electrode, not just in a columnlike path under your electrodes.

The orientation of the neurons in the cortical sheet relative to the stimulating electrodes is important. Neurons oriented as characterized in Fig. 2 will have excitatory effects under the anodal electrode and inhibitory effects under the cathode. In contrast, in tissue in which the neurons are inverted (e.g., due to cortical folding) the prototypical pattern of excitation and inhibition typically associated with anodal and cathodal stimulation will flip (Creutzfeldt et al., 1962). Intracranial recordings have suggested that intensity of the stimulation may differentially influence excitatory pyramidal cells and inhibitory interneurons, with evidence suggesting that stronger electric fields influence the excitability of pyramidal cells and weaker fields influence smaller inhibitory interneurons (Purpura \& McMurtry, 1965). However, it is far from clear that tDCS at the scale used with human participants has such selectivity on different cell types.

Although ionic gradients are clearly important in brining about the tDCS effects we observe, they cannot explain why tDCS effects last for minutes and even sometimes hours after the electrical field is turned off. If charged ions were the only mechanism underlying tDCS, then the brain would return to its normal state quickly due to diffusion in the extracellular space (Syková \& Nicholson, 2008). Instead, the electrical field must also be changing how the cells are communicating and functioning.

Researchers have sought to understand the relationship between the electrical field that tDCS generates and a variety of neurotransmitter systems. Glutamate has been clearly shown to play an important part in tDCS effects, with application of NMDA glutamate receptor antagonists modulating the effects of tDCS (Nitsche et al., 2003b; Nitsche et al., 2004). Similar types of pharmacological manipulations have implicated the dopaminergic system (Fresnoza et al., 2014a,b), and there is evidence that tDCS causes short-term changes of the voltage gated ion channels, which is probably less surprising (Liebetanz et al., 2002). Cholinergic, serotonergic, and GABAergic systems have all been shown to be related to tDCS effects (Medeiros et al., 2012). Finally, recent slice work indicates that the long lasting after-effects of direct-current stimulation appears to be due to a molecular cascade involving metabotropic glutamate receptors, NMDA receptors, and GABA that induce long-term depression and potentiation (Sun et al., 2016). In sum, essentially every signaling pathway has been implicated in the molecular and cellular activity that gives rise to the effects we observe with tDCS delivered to human participants. It is safe to assume that the biological basis of tDCS is not due to a single receptor type, neurotransmitter system, cell type, or other highly selective influence. It is also clear that we continue to determine the biological basis at the same time that we are testing its potential as a tool for the cognitive scientist and a treatment for the clinician.

\section{tDCS is bipolar}

The fact that tDCS creates an electrical field using both a cathode and an anode is a blessing and a curse. Being cognizant of the yin-and-yang of this circuit is of vital importance when designing your tDCS experiments. Previously we discussed the idea that the cathode and anode push and pull ions in the brain with opposite polarities. But what does this mean for brain activity? 
In our minds, the single greatest advantage of using tDCS as a tool is that the cathode and anode can have opposite effects on brain activity. This was shown in a series of foundational experiments using animal models. Bindman and colleagues recorded from anesthetized rats before and after they passed direct current through the brain tissue (Bindman et al., 1962, 1964). Some of their basic findings are shown in Fig. 3. The activity recorded from the rats' brains exhibited the normal baseline firing rates prior to any stimulation (Fig. 3 top panel). After several tens of milliseconds of stimulation, the brain activity near the cathode was significantly reduced (Fig. 3 middle panel). This is seen in terms of the number and size of spikes measured each second shown in the oscilloscope traces with the vertical tick marks (i.e., compare the middle panel of Fig. 3 with the top panel), and in the magnitude of multiunit activity shown in the thickness of the horizontal line on their oscilloscope traces. In contrast to this marked decrease by the cathodal electrode, brain recordings made near the anodal electrode showed the opposite effects (Fig. 3 bottom panel). These recordings show that the anodal and cathodal electrodes cause changes in brain activity in the opposite direction. The cathode decreases spike amplitudes and the likelihood of firing, while the anode increases spike amplitude and the likelihood of firing action potentials.

It is important to note that transcranial direct-current stimulation does not cause neurons to fire directly. Instead, it is believed that these changes in spike rates are because the voltage gradients bring neurons closer (following anodal stimulation) or farther (following cathodal stimulation) from their threshold for firing an action potential (Stagg \& Nitsche, 2011). This means that spikes are more or less likely following stimulation, and that this modulation can increase spontaneous firing rates due to inherent white noise in the system. It is also important to note that the direct-current stimulation of Bindman and colleagues was applied by placing electrodes directly on the brain itself. In other words, it was DCS, but not $t$ (transcranial) DCS. With human tDCS, if we apply 2 milli amps (2,000 micro amps) to the scalp, then models indicate that the maximum electrical field from this $2 \mathrm{~mA}$ is 0.4 volts per meter (Radman et al., 2009). Given this $0.4 \mathrm{~V} / \mathrm{m} * 0.3$ polarization per $\mathrm{V} / \mathrm{m}$, we can calculate a polarization maximum of $0.12 \mathrm{mV}$ at the soma, close to the axon hillock where action potentials are triggered. However, $0.12 \mathrm{mV}$ is not enough to depolarize a cell by itself. Thus, tDCS cannot cause cells to fire, unlike TMS that can induce an order of magnitude higher activity, and must work through neuromodulation and the build up of ionic gradients to nudge cells into a different state of functioning by changing membrane potentials.

The foundational work of Bindman and colleagues also showed the bi-directional effects on the neural activity elicited by stimuli. As shown in Fig. 4, when the forepaw of the rats received mechanical tactile stimulation, this elicited higher spike rates and larger amplitude local-field potentials following anodal stimulation compared to measurements during baseline periods. When they stimulated a second time, represented by the vertical lines in Fig. 4, they saw another increase in the firing rates elicited by the forepaw stimulation. Again, cathodal stimulation showed the opposite effects.

The basic observations that one of the electrodes increases activity and sensitivity of the cortex and the other decreases activity and sensitivity make tDCS a truly amazing tool with which to study perception, attention, and cognitive

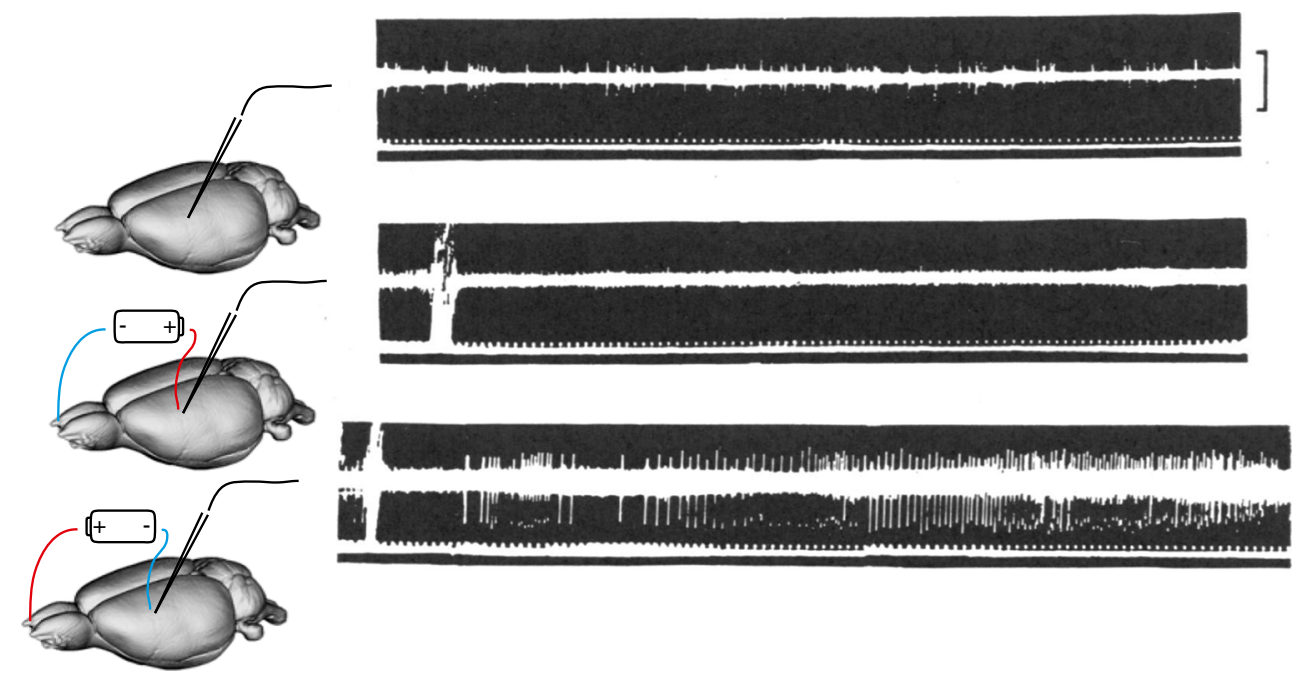

Fig. 3 The raw oscilloscope results from Bindman et al. (1964). The top trace shows the baseline recording from S1 of an anesthetized rat. Each spike is seen as vertical tick mark on the background hash of the multiunit activity. The dots at the bottom of the output show $10 \mathrm{~ms}$ tick marks. The voltage bar shows $10 \mathrm{mV}$. The middle trace shows activity at the same location after about $80 \mathrm{~ms}$ of cathodal stimulation. The bottom trace shows the activity after $20-30 \mathrm{~ms}$ of anodal stimulation 


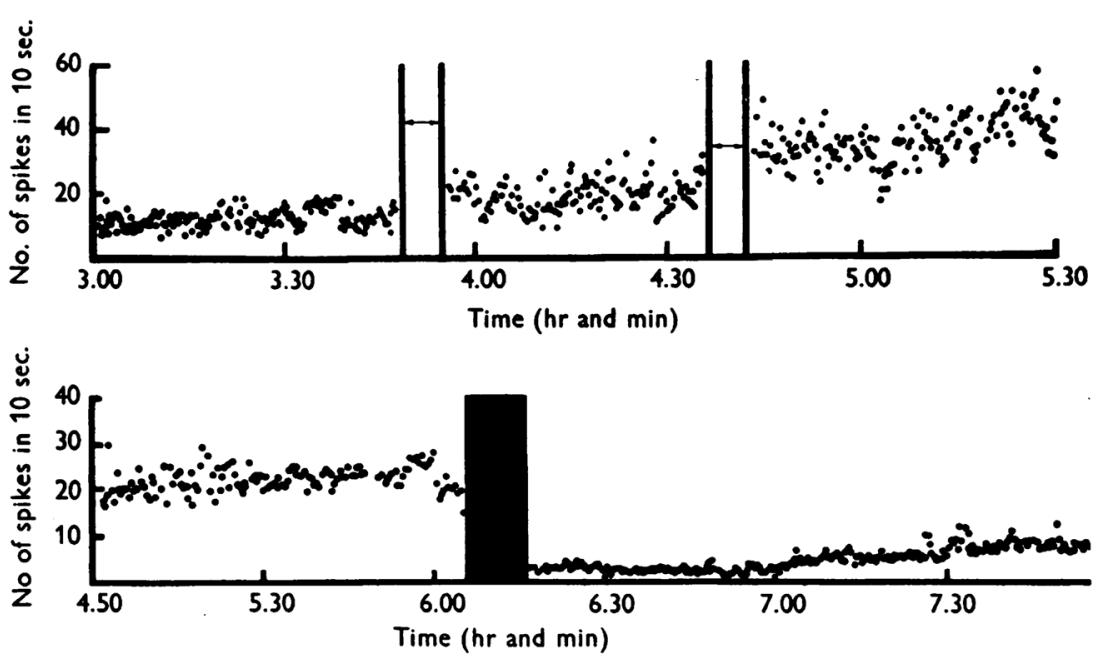

Fig. 4 The long-lasting firing rate effects from Bindman et al. (1964). The top trace shows the activity recorded from $\mathrm{S} 1$ of an anesthetized rat elicited by forepaw stimulation. The effects of about $8 \mathrm{~min}$ of low amplitude stimulation doubles the firing rate for $45 \mathrm{~min}$, another $8 \mathrm{~min}$ doubles the activity again. The bottom panel shows how cathodal stimulation shows the opposite effects with about $8 \mathrm{~min}$ of stimulation requiring hours before the firing rates normalize processing. The ability to have one knob that turns up activity and another knob that turns it down is not common in psychology and neuroscience. The closest method to the bidirectional causal manipulations of chunks of cortex that tDCS delivers are the currently evolving optogenetic techniques, and those are not ready for use in studies with humans.

In our work with tDCS, we have seen a number of cases in which we observe surprisingly symmetric effects of anodal versus cathodal electrodes. For example, we have seen anodal tDCS applied to medial-frontal electrode sites increase learning rates as participants learn a simple stimulus-response mapping, and those same learning rates decrease following cathodal stimulation (Reinhart \& Woodman, 2014). We have found a similar pattern of anodal stimulation resulting in improved function and cathodal stimulation impairing function when stimulating sites over visual cortex (Reinhart \& Woodman, 2015a; Reinhart et al., 2016). That is, anodal stimulation over posterior parietal cortex improved acuity and increased the amplitude of sensory event-related potentials (ERPs), whereas cathodal stimulation impaired acuity and decreased the amplitude of sensory ERPs. These are examples of cases in which $\mathrm{tDCS}$ of the human brain resulted in patterns similar to the direct-current stimulation effects observed by Bindman and colleagues in rats.

There are also ample observations in which only one direction of current flow has had an effect. Interestingly, it often seems to be anodal stimulation that changes behavior or brain activity, with the effects of cathodal stimulation being less consistent or reliable (Nozari et al., 2014; Santarnecchi et al., 2015). We have observed this in our own work. We found that when we stimulated lateral frontal cortex with anodal stimulation, it improved participants' ability to ignore a distracting stimulus (Cosman et al., 2015). However, cathodal stimulation did not change participants' ability to ignore these taskirrelevant distractors.

There are a few documented cases in which cathodal stimulation improves performance in a task, contrary to the intuitive expectations derived from the work of Bindman and colleagues. In one example, participants' with certain personality traits show improvement in a go/no-go task following cathodal stimulation of prefrontal cortex. Observations in which cathodal stimulation increases activity or improves task performance are surprisingly uncommon given the underlying neuroanatomy. If a chunk of cortex is on a gyrus versus the fundus of a sulcus, then the orientation of the excitatory pyramidal cells will flip relative to the surface of the skull (e.g., Clark et al., 1995). Currently, it appears that only approximately $10 \%$ of the human studies that have applied cathodal tDCS and tested cognitive functions have found that performance is improved in terms of reaction time (Santarnecchi et al., 2015), with accuracy improvements being exceedingly rare (Antal et al., 2001). Finally, there are cases in which both cathodal and anodal stimulation can impair performance relative to the sham baseline (Keshvari et al., 2013). This diversity of findings clearly points to our need to understand the underlying biophysical mechanisms of tDCS, as well as how the different parameters of these studies interact with the tissue being stimulated.

\section{Arrangement of tDCS electrodes given the bipolar electrical field}

The bipolar nature of tDCS, and electrical fields in general, emphasizes the importance of considering what we 
are doing when we are deciding where to put our tDCS electrodes. In experiments, we typically want to keep everything the same and just manipulate one variable. However, where we place our cathode or where we place our anode could be driving the effects that we believe are due to just one of these electrodes.

One common procedure in tDCS experiments is to place the anode over a location on one hemisphere and place the other electrode over the same location of the other hemisphere (e.g., Kadosh et al., 2010). Let us say we place the cathode over right prefrontal cortex and the anode over left prefrontal cortex. Assuming we are mostly changing the activity of large pyramidal cells, this means that we will be increasing the sensitivity of neurons in the left hemisphere and decreasing the sensitivity of neurons in the right hemisphere. So the effects could be due to either boosting up the left side or knocking down the right. But these two areas are heavily interconnected via the corpus collosum. So it could also be that any effects on information processing that we measure are due to an inability of left hemisphere to inhibit the right, or vice versa. This could be a useful way to understand hemispheric specialization. But if the goal is to understand the role of a brain area in a cognitive function, then the bilateral anodal-cathodal arrangement of electrodes will only show hemispherical asymmetries that exist and may not directly test typical network models in cognitive neuroscience (e.g., Corbetta \& Shulman, 2002; Serences \& Yantis, 2006). This situation is made more complicated in a region like prefrontal cortex in which the neurons have large receptive fields that often include the entire central visual field, or auditory surround (e.g., Schall, 1997), meaning that neurons in both hemispheres are likely to have overlapping receptive fields. For these reasons, it is rarely advisable to use an electrode configuration in which both the cathode and anode are placed on the same region of interest over the left and right hemisphere.

This leads to the question of where is the best place to put the other electrode (cathode or anode) when trying to target a region of interest. Although that is a simple question, there is no single simple answer for any area of the brain that you might want to hit with stimulation. The electrical field and the ionic gradient that tDCS produces will vary depending on the nature of the region that you want to target and your equipment. For these reasons, it is a good idea to create models of current flow prior to beginning an experiment. These models are useful in thinking about the nature of the bipolar electrical field you will be creating in the brain.

Several electrode locations have been heavily used when trying to pair an active electrode of interest with a second relatively indifferent electrode location. The configuration that we and other groups have used is to place one electrode over your region of interest and the other location on one of the cheeks (Berryhill et al., 2010; Hsu et al., 2011; Tseng et al., 2012). The cheek is a useful location because we can make a nice electrical contact. The only practical downside is that skin irritation due to contact with the sponges may be somewhat more common on the cheek compared to the scalp. The cheek is relatively near the brain, but the field induced by the electrode on the cheek is of maximum strength in the cheek, muscles, and gums, tissue that does not perform information processing. We often favor the right cheek as a default, although comparing results with both cheeks can be useful, as the field strength is often significant in the ventral lateral prefrontal cortex near the cheek being used. Figure 5 shows an example of how the left versus right cheek locations for a cathodal electrode will induce relatively high field strengths in the brain regions nearest those sites. Similar to the search for the perfect reference in human electrophysiology (Luck, 2005), people have tried many other locations, such as just above the left or right orbit (i.e., near the frontal pole). However, none of locations on the head, including the cheek, provide a perfect location without any influence on unintended brain regions. The fact that the other electrode might be the cause of your results is probably the primary weakness in tDCS experiments. Often this requires a control experiment with a different electrode configuration to rule out the explanation that the opposite polarity electrode is the cause of the tDCS effects observed.

The other consideration when configuring the locations of your tDCS electrodes is that some arrangements can have negative side effects. In some of our work, we have used bilateral electrodes paired with cheek electrodes that generate paths that converge in the area of the mouth. Participants often report a metallic taste in the mouth when we have used such arrays, especially at higher intensities. Fortunately, that taste is often as strong when sham stimulation is used, but this may induce a multisensory

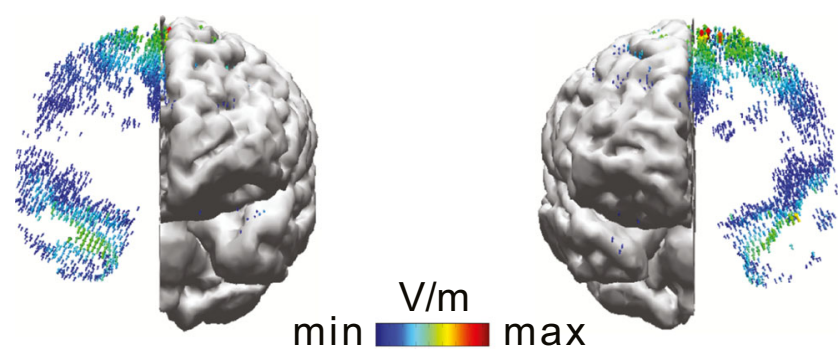

Fig. 5 Current flow models of a $19.25 \mathrm{~cm}^{2}$ anodal electrode over medialfrontal cortex (site $\mathrm{Fz}$ in the 10/20 system) and a $52 \mathrm{~cm}^{2}$ cathodal electrode on the right cheek (left panel) or left cheek (right panel) at $2.0 \mathrm{~mA}$. This figure shows how even the cheek sites are likely to produce fields in the lateral portions of the prefrontal and temporal lobes 
environment that is noxious to the subject. Your equipment manual and other sources do a good job of warning the experimenter against placing the electrodes on the neck and other nearby tissue ( Kuo \& Nitsche, 2012; Utz et al., 2010). Arrays that stimulate the brainstem circuitry can have negative effects on heart rate, breathing, and pain sensations. Generally keep the current path away from the neck and spinal cord. However, some groups use the upper arm relative to a location on the head and have found that it does not result in complications involving the brainstem or spine (e.g., Clark et al., 2012). This many be an attractive location for the other electrode in tDCS that does not put another cortical area in a region of high current intensity.

Some of the most advanced systems use higher density arrays of electrodes to inject current at one location and pull that current off from an array of nearby electrodes of the opposite polarity (or vice versa). An example of this is shown in Fig. 6. These can be arranged using spatial configurations that will remind cognitive psychologists of Duncker's radiation problem from the problem solving literature (Gick \& Holyoak, 1980), in which you divide your forces so as to minimally impact all but your target. That is, you can array a set of cathodes with a single anode to pull current through a specific region of the brain, even when that region is relatively distant from any particular electrode and without pulling excessive current through any of the regions near any particular cathode. These systems are sometime known as High-Definition tDCS (or HDtDCS) to communicate the greater density of stimulation elements with the goal of delivering stimulation with greater precision. This array of electrodes offers the ability to precisely target specific parts of the brain, although it can come at the cost of being more uncomfortable to the participant than simple two-electrode systems with large electrodes. But the bi-directional nature of tDCS could be a problem for these systems too. That is, with the simple configurations of these electrodes in which four cathodes ring an anode, you are increasing the activity in one part of the brain while decreasing the level of activity in the parts that spatially flank the target. Because connections between areas are highest for spatially nearby regions (Markov et al., 2014), this could result in a large degree of cancellation of the stimulation between neighboring patches of cortex. As it stands, the current flow models of HD-tDCS systems propose that it is possible to target a single cortical area with flanking electrodes that do not induce a flanking surround of the opposite polarity (e.g., Soterix Medical Inc., New York, NY, USA). However, fine scale neuroscientific experiments are needed to understand the spatial distributions of these more advanced systems.

Finally, we recommend that you use a method of electrode placement that enhances reproducibility. The simplest method that we have found is to use the $10 / 20$ system for electroencephalogram (EEG) electrode placement (Jasper, 1958). This is a system of placing electrodes on the scalp that is already universally used in cognitive neuroscience and is already used to integrate findings across studies (Santarnecchi et al., 2015). Elastic caps of a variety of sizes with the locations marked can be purchased from a variety of vendors fairly cheaply. A laboratory could also measure the electrode locations by hand with a cloth tape measure using this simple system, although this is more time consuming. An alternative to this method is to localize specific brain areas with structural imaging and then target these structures based on anatomical landmarks or even functional localize an area with neuroimaging. The method of functional localization is common with TMS (e.g., Neggers et al., 2004) and similarly useful with tDCS (Clark et al., 2012). Functional localizers can be especially beneficial for targeting regions of the brain

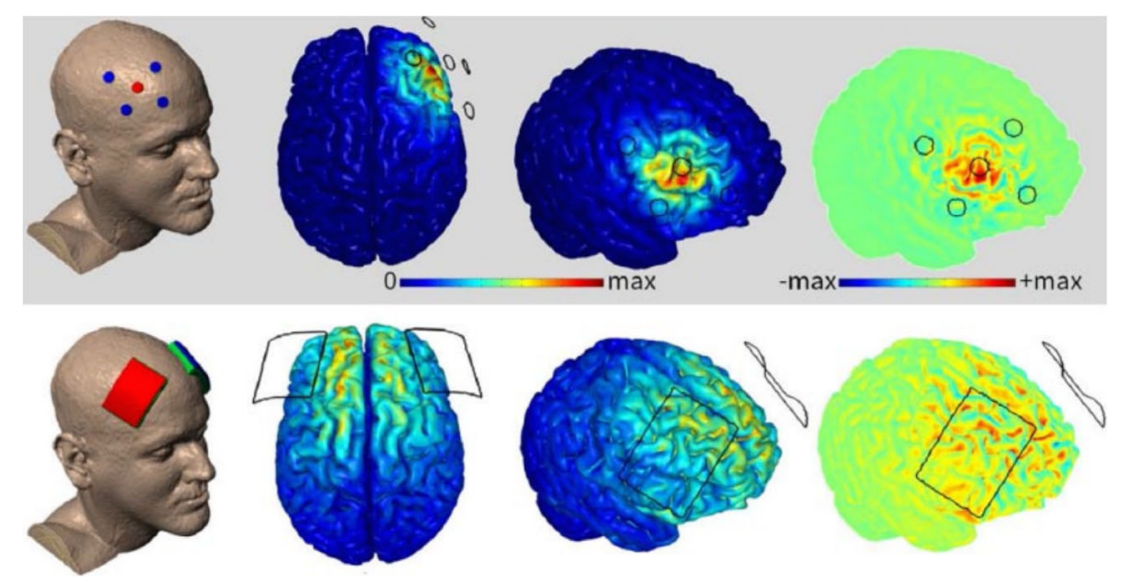

Fig. 6 Current-flow models targeting the dorsolateral prefrontal cortex using traditional $1 \times 1$ tDCS with two large sponge pads versus $4 \times 1$ high-definition tDCS (HD-tDCS) with five small ring electrodes. The models demonstrate the increased spatial resolution of HD-tDCS. Adapted with permission from Shekhawat et al. (2015) and Neurorehabilitation and Neural Repair 
whose locations can vary substantially across individuals (e.g., prefrontal cortex, see Van Essen \& Dierker, 2007). This method of localizing neural structures also has the advantage of specificity, assuming electrodes and current flow are capable of taking advantage of this precision, which may be presumptuous based on our current knowledge and methods. However, the use of the 10/20 system has the advantage of being the most accessible for researchers regardless of the availability of grant funds or imaging resources. Even if a functional localizer is used to target a region of the brain it enhances reproducibility to report what electrode in the 10/20 system the localized site was nearest.

\section{Safety and side effects}

In the last section, we discussed how you want to avoid certain regions of the central nervous system with your electrodes. The good news is that this is one of the only known safety concerns with tDCS (Bikson et al., 2016; Kuo \& Nitsche, 2012). Unlike TMS, which can induce seizures in some participants prone to such problems, tDCS results in no increase in such side effects (Bikson et al., 2016; Poreisz et al., 2007). This means that tDCS can be safely used without a medical professional onsite while participants are run in experiments.

The side effects that are felt are itching and tingling as tDCS is being delivered (Poreisz et al., 2007). Most people report that these are mild. At least one of us is overly sensitive and feels that the itching is fairly intense initially. The additional good news in this regard is that participants habituate to this fairly rapidly and the itching sensations fade quickly. It seems that the itching and tingling sensations may be due to the change in voltage as it ramps up and down at the beginning of the delivery of stimulation.

Some participants report moderate fatigue, particularly during, but not after stimulation of motor cortex in a large sample of experiments (Poreisz et al., 2007). This does not seem to happen with stimulation of other brain areas. It also seems to be consistent with what we might expect given the nature of the cortex being stimulated. We have not found that this side effect is reported at a higher rate than the sham baseline when stimulating medial prefrontal, lateral prefrontal, parietal, or occipital cortices. To determine whether any of our participants experience side effects following tDCS stimulation or the sham we use debriefing questionnaires, adapted from others (Gandiga et al., 2006; Poreisz et al., 2007). We provide these questionnaires in Appendix A.

The body of work that has looked at safety of tDCS has generally proposed that total charge density to the skin surface of the scalp should not exceed 960 coulombs per meter squared $\left(\mathrm{C} / \mathrm{m}^{2}\right)$ (Bikson et al., 2016; Iyer et al., 2005; Nitsche \& Paulus, 2001, Nitsche et al., 2003a; Nitsche et al., 2003c). The most widely used tDCS protocols generating charge densities at or below this threshold include relatively large wet sponge pads (i.e., $25-35 \mathrm{~cm}^{2}$ ), current intensity ranging from 1 to $2 \mathrm{~mA}$, and a continuous application time of up to $20 \mathrm{~min}$. Other factors that can influence charge density are sponge salinity, electrode configurations, and ramp waveform. The tDCS protocols operating within these limits are generally considered safe based on experiments showing no increase in negative side effects (such as changes in tissue volumes, pain, etc.) with only isolated reports of minor skin irritation under the sponges (Bikson et al., 2016; Dundas et al., 2007b; Iyer et al., 2005; Nitsche \& Paulus, 2001; Nitsche et al., 2003a; Nitsche et al., 2003c; Poreisz et al., 2007). However, screening out participants who self report having sensitive skin can minimize the risk of skin irritation. This work also showed no negative side effects when measuring tissue volume with MRI, or changes in the levels of the stress hormone cortisol (Raimundo et al., 2012). It is possible that even higher stimulation settings could be safe if we base our conclusions on the animal models (Liebetanz et al., 2009), however, additional work is needed to investigate the thresholds and biophysical mechanisms of tDCS to verify that possibility before using such intensities in human participants.

\section{tDCS can last a long time}

The work of Bindman and colleagues highlighted another powerful aspect of this type of brain stimulation: its long duration. Bindman and colleagues (Bindman et al., 1964) noted that they needed to be careful with how long they passed current through the brain tissue. This was not because it would damage the brain with the low amperage they were using, but because the effects would last for more hours than the experimenters could tolerate. When passing current for approximately $20 \mathrm{~min}$, they noted that even after $5 \mathrm{~h}$ the anodal effects on brain activity remained, resulting in their decision to abandon their efforts to determine the total duration of the stimulation in their anaesthetized animals. This can be taxing for experimenters who are stuck on the bench during these experiments. However, the long-lasting nature of direct-current stimulation is ideal for scientists seeking to use it to understand brain functions during tasks that require many trials to obtain reliable measures, or clinicians seeking to treat brain disorders.

In experiments with humans using tDCS, we have not yet seen effects that last beyond our ability to measure. Instead, early work stimulating motor cortex suggested that $\sim 1.5 \mathrm{~h}$ was the limit (Bindman et al., 1964; Nitsche 
\& Paulus, 2001). More recent work with stimulation targeting frontal and prefrontal cortex suggests that effects on behavior and brain activity can last up to $5 \mathrm{~h}$ (Reinhart \& Woodman, 2014). It is safe to say that we do not yet know what the duration of effects will be when a given area is stimulated, but that hours appears to be the appropriate unit of analysis.

Several factors will determine how long a tDCS effect can last. The first factors are the duration and intensity of the stimulation. Longer stimulation protocols with higher amperage will have longer effects (Nitsche \& Paulus, 2001).

The third factor that is likely to be important is the nature of the brain structure being targeted. It appears that the relatively machine-like areas of motor and visual cortex have effects that last about $1.5 \mathrm{~h}$ following a single session of stimulation lasting 20 min (see Fig. 7A) (Nitsche \& Paulus, 2001; Reinhart et al., 2016). In contrast, the more delicate less machine-like structures in frontal and prefrontal cortex that carry out higher-level computations may be easier to perturb for longer intervals (see Fig. 7B) (Reinhart \& Woodman, 2014, 2015a).

The fourth factor that will determine stimulation duration is that some cognitive functions might simply be so heavily distributed that it may be difficult to influence their function. Our group, and a number of others, tried to change visual working memory capacity (i.e., the number of simple objects people can remember) by stimulating prefrontal cortex with tDCS. Someone may yet discover how to do this, but we know of many groups who have tried various approaches without luck. It is possible that this cognitive function is sufficiently distributed so as to not be easily perturbed with tDCS.

The fifth factor that we know is important for the duration of effective tDCS is the folding patterns of the cortex underlying the electrode. The orientation of the cortex and the pyramidal cells in it have long been known to change the nature of the electrical fields that are generated from within the tissue during its normal operation (Luck, 2005). These same factors will be important when an external electrical field is applied to this tissue (Creutzfeldt et al., 1962).

Sixth, is it possible to give several doses of tDCS and see a super-additive increase on the duration of the tDCS effects? That is, perhaps one 20-min tDCS session lasts for $5 \mathrm{~h}$, but two sessions on the same day could last for 3 days or more. This is the dream of many ERP researchers seeking to improve psychiatric disorders with tDCS (Reinhart et al., 2015). Repeated stimulation spaced across time is a method that many laboratories are examining as a way to instantiate long-lasting effects (Liu et al., 2016; Nitsche et al., 2008). Some work with animals suggests that multiple sessions can have a cummulative effect (Rueger et al., 2012; Rushmore et al., 2013). A considerable effort is being made with human participants to determine whether tDCS effects are more potent with multi-session tDCS (Dumel et al., 2016; Galvez et al., 2013). This work is ongoing given the difficulty in designing the proper control groups (Boot et al., 2013), but there is great hope that the duration of tDCS after effects could be significantly increased with repeated exposure (Alonzo et al., 2012; Paneri et al., 2016).

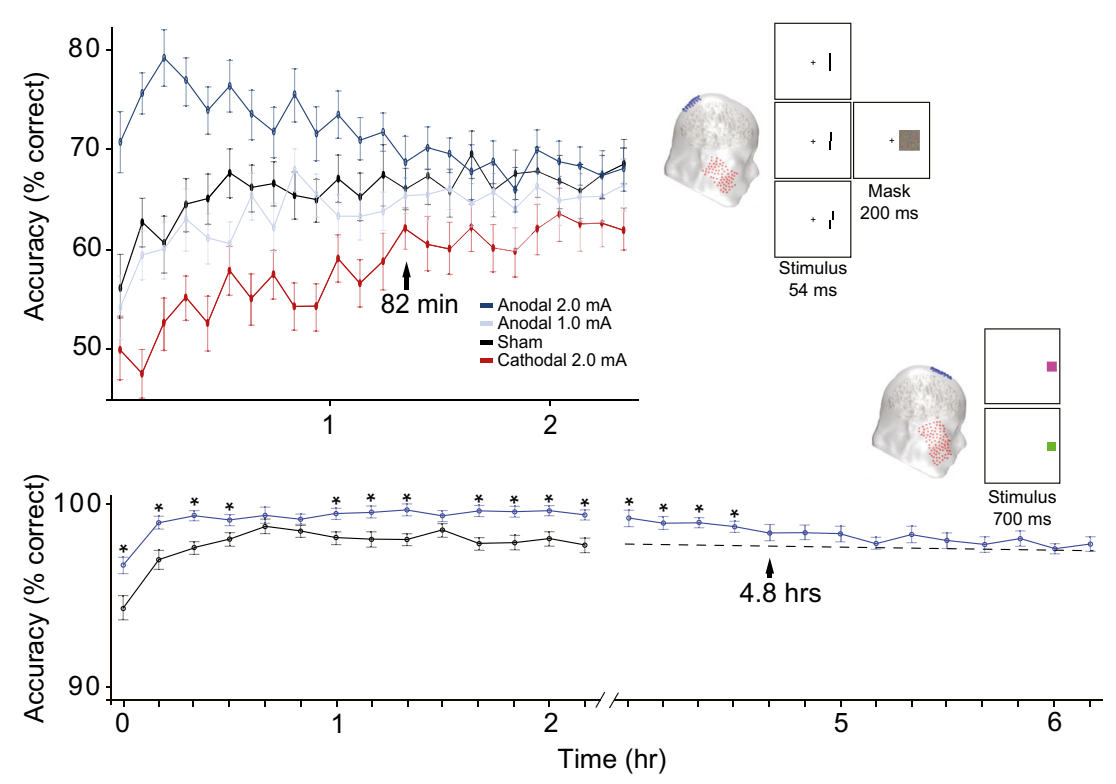

Fig. 7 Duration of transcranial direct-current stimulation effects during a vernier acuity task (top) and a task where observers learned stimulusresponse mappings with stop signals interleaved (bottom). In the experiments show in the top panel stimulation was applied to posterior parietal cortex (electrode site P3) for $20 \mathrm{~min}$ at $2.0 \mathrm{~mA}$. In the bottom panel it was applied to medial-frontal cortex (Fz) for 20 min at $2.0 \mathrm{~mA}$. Adapted with permission of the Cell Press and Society for Neuroscience from Reinhart et al. (2016) and Reinhart and Woodman (2014) 
The final factor that determines the duration of tDCS effects is the task that participants perform during or following stimulation. If participants are learning or storing new information in memory, then it seems reasonable to propose that changes could last as long as human memory is capable (Kadosh et al., 2010).

How is it possible that several minutes of stimulation can have effects that change the brain for hours? This has been an interesting question that the early neurophysiologists who pioneered the use of direct-current stimulation asked. An early study tested the hypothesis that these long-lasting effects were due to direct current eliciting structural changes in how neurons were wired together. Gartside (1968) recorded neural activity from anaesthetized rats. He first applied a variety of drugs topically to the surface of the brain. Then $30 \mathrm{~min}$ later, he applied anodal stimulation to the cortex for $10 \mathrm{~min}$. In the no-drug baseline, the elevated activity measured during the stimulation continued after the stimulation was switched off. However, when drugs were applied that prevented protein synthesis, the after-effects were abolished, such that the firing rates returned to baseline immediately after the stimulator was switched off. These findings show that long-lasting effects of tDCS require protein synthesis to be occurring during the delivery of stimulation. When drugs were applied immediately after the stimulation, there was no reduction in the elevated firing rate. Thus, it was concluded that direct-current stimulation may be having its long lasting after-effects due to changes in the connections between neurons, like long-term potentiation (LTP) or long-term depression (LTD) are known to induce. More recent slice work appears to be consistent with this notion (Sun et al., 2016).

It may be obvious after reading about these effects that performing a task during stimulation delivery may be particularly effective because it takes advantage of these LTP-like effects as stimulation is delivered. Reis and Fritsch (2011) suggest that stimulation is most effective when delivered during the processing of task-relevant information. However, there is also evidence that performance is most improved after stimulation is over (Barbieri et al., 2016). More data are needed to determine whether performing tasks during stimulation results in longer lasting effects of tDCS.

\section{Modeling the electrical field}

Models of the tDCS current flow are useful for targeting specific brain regions with tDCS. Moreover, these models aid our thinking about whether our effects of interest are due to the anodal or cathodal end of the electrical field. These models are also helpful in providing potential targets for investigation by researchers using techniques that afford greater spatial precision, including neuroimaging and invasive neurophysiology. Researchers have also proposed that a great need for tDCS research is to more frequently and precisely model the electrical fields that stimulation induces (Parkin et al., 2014).

In this section we will provide specific technical details about how to model the electrical fields induced in the tissue during tDCS experiments. We provide pointers to software we have found useful and citations to the hard work that has guided our modeling efforts. Because of the nature of this material, we heavily rely on the terminology of physics and neuroanatomy to deliver the details in a concise way. The potential user of these methods should read the documentation for the software and the citations we provide to fully understand what they are doing.

There are several important things to keep in mind about simulations of tDCS current flow. First, our understanding of the electrical fields generated by tDCS continues to evolve. A good example of this are the recent recordings and the modeling both showing that the strongest electrical field gradients are generated parallel to the surface of the brain and perpendicular to the surface of the skull (Rahman et al., 2013). This is an important observation because in the human brain, with its highly folded structure that varies across individuals, the precise regions that are most affected by tDCS will vary as the folding patterns vary across people.

Second, much of what we know from modeling how tDCS affects the cortex is based on models of the electrical fields generated by the cortex itself, and recorded using the EEG. What we know from this work is that it is important to base these models on as much information about the brain as possible. For example, the most accurate models of electrical activity in the human brain use structural imaging data, realistic volumetric head geometries, and finite-element methods (Bikson et al., 2012; De Lucia et al., 2007; Sadleir et al., 2010; Wagner et al., 2007). Ideally, customized current-flow models would be constructed based on individual neuroimaging data to overcome many of the individual differences in the brain such as cortical folding, skull thickness, and head fat.

Figure 8 summarizes the steps for calculating a currentflow model. We will describe each of these steps with pointers to the relevant software and citations that you would need to compute your own tDCS models.

\section{Creating a model of the head using structural images}

The first step in creating a model of the electrical field produced by tDCS is to create a model of the tissues in the head. This is done with structural magnetic resonance imaging (MRI). Advanced users can generate their own realistic head models using individual participants' structural MRI data. Research shows that individualized modeling can increase the accuracy of stimulation intensity for a region of interest 


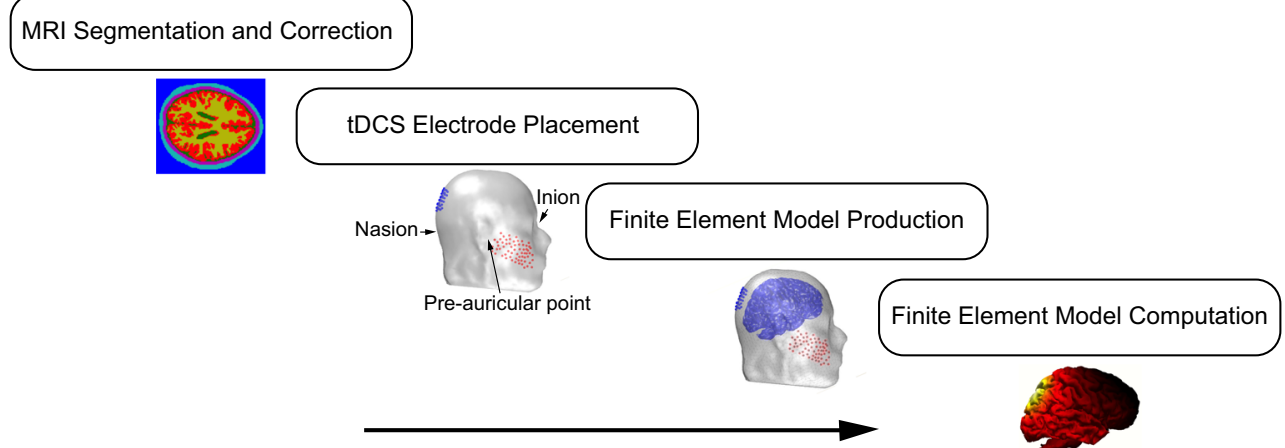

Fig. 8 Computational workflow for traditional tDCS modeling. The steps for modeling tDCS current flow commonly include MRI segmentation and morphological correction (Matlab, SPM8), the placement of tDCS electrodes (Matlab, ScanIP, FieldTrip), finite element model generation or meshing (CURRY, ScanIP), and finite element model computation (SCIRun, Abaqus)

manually using ScanIP (Simpleware, Exeter, UK), specifically in the ScanCAD Module. Open source electrode placement tools are also available (e.g., FieldTrip) though often involve manual changes through an interactive graphical user interface. The coordinate system of tDCS electrodes is typically the EEG International 10/20 (or 10/10) System, defined in terms of anatomical landmarks on the outside of the head, including the nasion, inion, and the left and right pre-auricular points. To transform the EEG coordinate system to the Right Anterior Superior (RAS) orientation of the MRI volume, three unit vectors must be defined from the nasion to the inion, the pre-auricular left to the pre-auricular right, and the class product of these two vectors. Fiducial points for extraencephic tDCS electrode placement are often provided by the user through visual inspection using various interactive 3D viewers (e.g., FieldTrip, MRIcro 3D, http://www.mccauslandcenter.sc.edu/crnl/mricro).

\section{Finite element model production}

The next step of current-flow modeling is the production of a finite element model. The different tissue masks make up the volume conductor model of the head. Software such as CURRY or ScanIP can be used to obtain a tetrahedral tessellation of the head in which all elements correspond to exactly one tissue type. This process of space filling or tiling in 3D Euclidian space, results in a large mesh of cells that consists of hundreds of thousands of vertices and tetrahedra. In brief, the production of meshes is a process where each mask is divided into small contiguous elements, which allow the current flow to then be numerically computed. The isotropic electrical conductivity values assigned to each tissue compartment are often provided as a default setting in many software packages that build volume conductor models. For example, in CURRY the segmented compartments and their respective isotropic electrical conductivities (in $\mathrm{S} / \mathrm{m}$ ) include: skin (0.33), skull (0.0042), and brain (0.33). However, these values can also 
be manually entered to ensure consistency with standard estimates from the literature based on in vivo and in vitro measurements (e.g., Akhtari et al., 2002; Baumann et al., 1997; Dannhauer et al., 2011; Haueisen et al., 1997; Oostendorp et al., 2000). For improved results, you can obtain diffusion tensor imaging (DTI) data from participants, then model gray and white matter as anisotropy conductivities from the registered DTI using an effective medium approach (Tuch et al., 2001).

\section{Finite element model computation}

After the finite element model is generated, simulation of the distribution of current inside the head is achieved by solving the Laplace Equation. This can be done with several different software packages, such as Abaqus (SIMULIA, Providence, RI, USA) or the freely available package under MIT license called SCIRun (http://software.sci.utah.edu). This software uses the quasi-static approximation of Maxwell's equations, which results in the Laplace equation. That is, because there is no source from our tDCS inside the volume conductor, the potential inside the head follows the Laplace equation. The tDCS forward model is regarded as a mixed boundary value problem because the electrical potential at the location of the tDCS electrodes is determined by the Dirichlet (or first-type) boundary conditions, whereas potential at all other external locations on the surface is determined with inhomogeneous Neumann (or second-type) boundary conditions. For bipolar electrode configurations, the modeled electrodes located at the region of interest on the head have the potential set to the appropriate stimulation intensity with all other locations at the external surface treated as insulated.

Mathematically, forward models of tDCS current flow are well posed problems with a unique solution, unlike inverse source modeling of electrical activity generated by the brain itself (Luck, 2005; Woodman, 2010). Although the steps in tDCS forward modeling are fundamentally the same across publications and laboratories, small differences in modeling procedures can result in meaningful differences across current-flow solutions. Thus, it is important to stress that a tDCS model serves as a working hypothesis for where the electrical field travels through the brain given the tDCS parameters used.

A number of vendors of tDCS equipment will provide software to aid your modeling of current flow in the brain. There are several pieces of software that allow users with little to no experience with computer modeling to generate current-flow simulations using intuitive graphic user interfaces and unlimited hands-on customer support. Software from Soterix Medical Inc. (New York, NY, USA) is a case in point, providing tools to allow users to explore model solutions with considerable flexibility. For example, users can choose among a variety of different pre-loaded adult heads, including the Standard MNI 152 head, or create a custom model by using their own participant's MRI data. Users can experiment with different electrode shapes, sizes, and positions on the head, and target brain areas by name to reverse generate an optimal tDCS montage based on maximum focality or intensity of current flow.

\section{The surprising effectiveness of sham stimulation}

So far in this tutorial we have discussed the effects of applying stimulation for protracted periods of time and how to model the resulting electrical field. However, tDCS is also special in terms of the effectiveness of its sham conditions. Sham stimulation using TMS is difficult because the sound and somatosensory stimulation that pulses cause, similar to a thump on the skull with someone's finger. This makes their absence easy to detect in a baseline condition. With tDCS, participants' ability to distinguish between sham and active stimulation conditions is often no different from chance, as we will describe next.

The typical protocol for sham stimulation with tDCS is to ramp the stimulator on and then off during the beginning of a period that matches the period used with active stimulation, and then ramp it on and off again at the end of that period. For example, we often stimulate for $20 \mathrm{~min}$. In the sham condition, we ramp the stimulator on, leave it on for $10 \mathrm{~s}$, and then ramp it off. After 20 min we repeat that same procedure (see the top panel of Fig. 9).

At the end of each stimulation session, whether anodal, cathodal, or sham, we ask participants to guess whether the stimulator was on continuously and whether current flowed toward the cheek or away from it. We find participants are at chance at making this discrimination (Reinhart \& Woodman, 2014, 2015a). This means that tDCS experiments can easily be run using withinsubjects designs because experience with one condition cannot be distinguished from another condition. In one case we found that people appeared to be slightly worse than chance because they thought that the sham stimulation condition is actually the anodal stimulation condition (Reinhart \& Woodman, 2015a). It appears that turning on and off of the stimulator in the sham condition may result in more of the itching and tingling sensations than actual stimulation. This results in some participants believing that the stimulator is doing more during the sham condition making it difficult to distinguish between active and sham conditions.

The slight bias to believe that sham conditions are delivering electrical stimulation has an interesting implication. It is likely that demand characteristics, arousal, and other nonspecific factors actually work against tDCS 

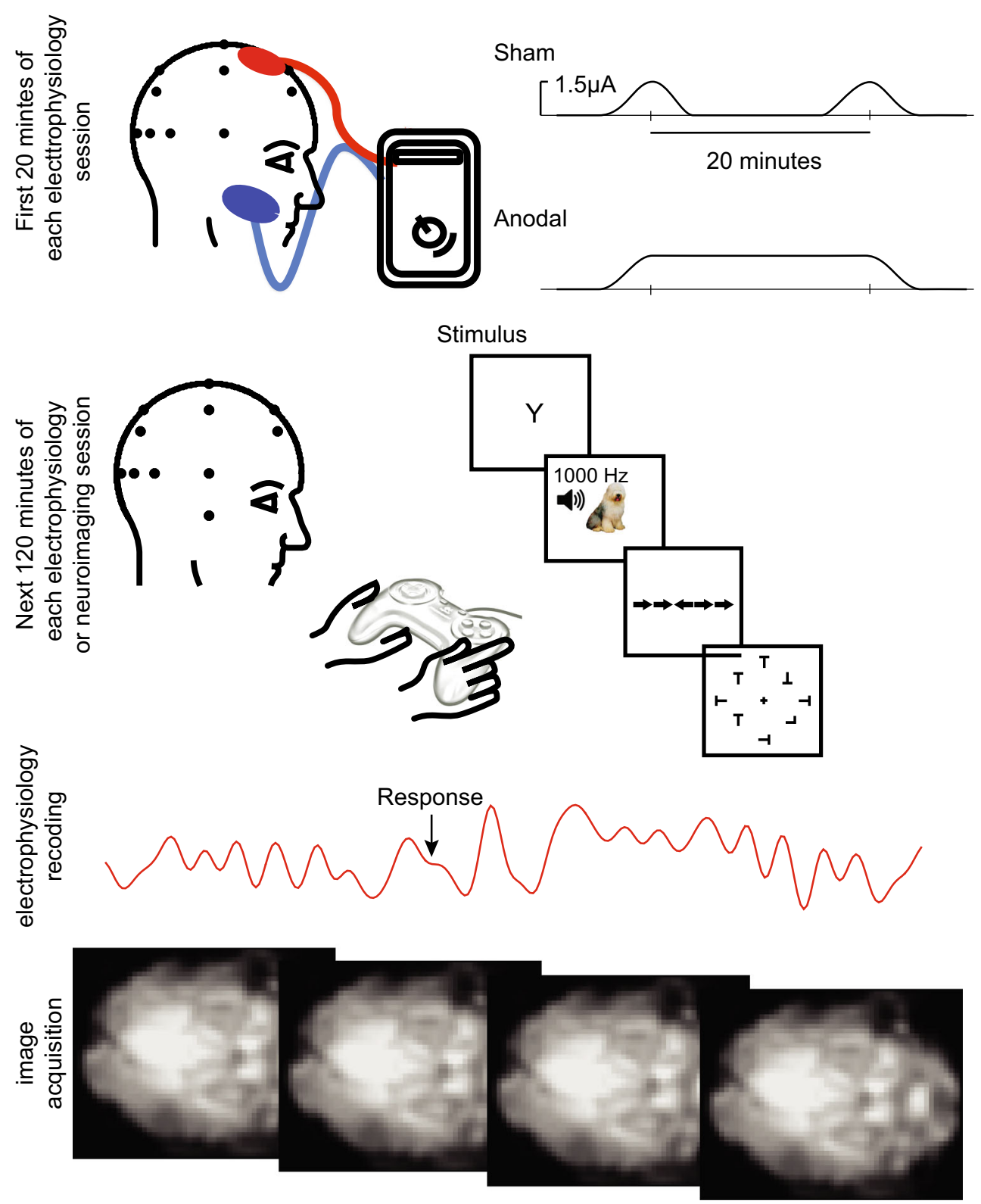

Fig. 9 An example of a 20-min application of sham stimulation (top panel) and active anodal stimulation (second panel down). After the application of tDCS, the long-lasting after effects are sufficient that alternated brain activity and cognitive processing can be measured for 1$5 \mathrm{~h}$ in many stimulation protocols and sites. The bottom panels illustrate

experiments finding a significant difference between active and sham stimulation conditions. That is, an active stimulation condition that results in improvement of performance has to work against participants' bias that sham is the real stimulation session. These expectations in the sham condition likely result in heightened arousal that boosts performance relative to what we would measure if the task were performed in a true baseline without any electrodes attached to the participants. This also points to the utility of using both anodal and cathodal stimulation conditions to compare with sham. It is more difficult to that tDCS easily allows for the recording of electrophysiological responses or the acquisition of neuroimaging data following stimulation. It is also possible to acquire data during stimulation with certain stimulators and recording equipment

explain a bi-directional pattern of results with a simple influence like arousal.

The take away message is that with tDCS you should always try to use within-subjects designs. Within-subjects designs are superior because they remove subject-wise variance from the effect of stimulation that you want to measure. Given that participants are blind to whether they are in the sham or active stimulation conditions, the sham condition provides an excellent control condition. There are situations in which within-subjects designs are not possible, such as training studies. In these cases larger samples of subjects are needed to 
overcome the increased variance in the absence of the withinsubject manipulation.

\section{A typical tDCS experiment}

Generally researchers new to the tDCS technique will struggle to conduct effective experiments because there are no standardized procedures or strict principles for how to set up a successful tDCS protocol. Here, we will provide a concrete example of a tDCS experiment. Although many of the parameters used in tDCS experiments have yet to be systematically evaluated, we hope a thorough description of our methods will be useful for researchers new to the technique, as well as for illustrating general procedures and common problems. The experiment we will describe was conducted to understand visual perception. We will describe an experiment using a conventional two-electrode stimulator (i.e., a $1 \times 1$ montage), however, many of the steps are similar regardless of the stimulation montage you might use.

As always, the first step is to obtain consent. Our consent form describes that the electrical stimulation can result in effects can last up to $5 \mathrm{~h}$. It describes the common itching and tingling that people feel when the stimulator is on. We tell the subject that the device delivers current using a 9-volt battery and that this is similar to a scientific application of the type of current they may have felt when putting their tongue on a 9-volt battery in the reckless days of their youth.

Next, we position our stimulating electrodes. In most of our experiments, we position an elastic cap with the 10/20 electrode positions marked. This helps us make sure we are placing the stimulating electrodes at the same locations for each subject. We have multiple sizes of elastic caps that ensure that the electrode locations are placed correctly for the size of the individual's head.

After marking the stimulation site with the cap, we then place a wet sponge between the conductive rubber electrode and the surface of the skin. These sponges have been soaked in salt water or electrolyte fluid to increase the conductivity of the sponge. There is a goldilocks amount of saturation for these sponges, with too much or too little causing problems, so we discuss this point in detail next.

If you do not wet your sponge contacts enough, you will not have a sufficiently low resistance connection and this is needed to complete the circuit. Most stimulators check the quality of the circuit as stimulation is delivered and notify the user if resistance gets too high. So sponges that are too dry are typically diagnosed by your equipment.

Sponges that are too wet are actually a bigger problem. If the sponges are too wet, water that leaks off of a sponge when it is pressed against the skin can spread to the other electrode and create an electrical connection between the two electrodes. This produces a short circuit. The current follows the path of least resistance through the water and wet hair connecting the two electrodes and not through the skin, skull, and brain. Experiments show that under ideal circumstances, approximately $45 \%$ of the current actually goes through the brain (Rush \& Driscoll, 1968). By having extremely wet sponges, this can quickly change to $0 \%$. In electrophysiological recordings this is known as a salt bridge that can connect two electrodes and result in their becoming one super electrode that hurts spatial resolution (Luck, 2005). When this happens in tDCS experiments it means that potentially no stimulation is being delivered. Excessively wet sponges can also cause the tDCS electrodes to shift across the head during stimulation. We recommend having sponge pads just moist enough to complete the circuit between the subject and the stimulator. With this approach, the sponge pads may dehydrate over time changing the total charge density or breaking the circuit connection. To avoid this, simply use a pipette or syringe to add more salt solution to remoisten the sponge pads if you observe a decrease current level during the course of the experiment.

The salt solution used to moisten the tDCS sponges is also an important consideration in connection with subject comfort and the total amount of charge delivered to the brain (Dundas et al., 2007a). Generally, solutions with lower sodium chloride concentrations are better tolerated by participants. Because ionic strength of deionized water is weaker than any sodium chloride solution, more voltage is needed to deliver electrical current through the skin, skull, and brain when salt solutions are weak. In our experience, to assure subject comfort and minimize stimulation voltages, we have found a salt solution toward the lower end of the typical concentration range of 15$220 \mathrm{mM}$ to be most successful. For reference, the upper end of this range includes the $0.9 \%$ saline $(\mathrm{NaCl})$ solution known as normal or physiological saline because it has the same osmolarity as plasma $(0.9 \%$ saline is equal to $154 \mathrm{mM}$ ). Relatively low salt solution is also beneficial because it reduces local vasodilation (i.e., skin redness) at the sites of stimulation, which correspondingly reduces the possibility of an experimenter unblinding him- or herself to the nature of the stimulation.

So now you have the electrodes placed with good contacts and just the right amount of water or conductive medium to not create problems. Now you need to either apply stimulation or perform a sham condition. For obvious reasons, it is ideal if this can involve a twoexperimenter procedure in which the experimenter who works with the subject is blind to whether sham or active stimulation is being delivered. Then, the stimulator 
experimenter comes in only to run the stimulator. For this type of double-blind procedure, you can have the experimenter who will run the subject through the subsequent task and analyze the data leave the room before stimulation is delivered. Some tDCS equipment provide arbitrary numbered buttons that trigger the different stimulation conditions so that a single experimenter is not aware of whether stimulation is being delivered on a given session. In this case, only one experimenter need be present when running the subject.

Blinding the subject is typically easier than blinding the experimenter due to the nature of the sham sensations, and the small size of these devices. In our work, we have removed the stimulator controls from view by having the stimulator control module placed behind the subject when using unsophisticated types of stimulators that have lights indicating when the unit is continuously on. This way they cannot see if lights are illuminated during the entire 20-min period.

It is natural to ask, how many subjects will I need to run? As other researchers have recently noted (Berryhill et al., 2014), a number of the early tDCS papers that examined the modulation of cognitive processing used fairly small samples (i.e., approximately ten subjects). However, positive results that have appeared more recently in the literature typically involve samples that are double those initial papers. It is important to keep in mind that what we are typically looking for is an interaction of stimulation condition with a cognitive manipulation of interest. For example, we might be varying the stimulus set size across trials and determining whether stimulation interacts with that parametric manipulation of set size. Obviously, measuring an interaction of stimulation condition (e.g., anodal, cathodal, and sham) with set size (e.g., 2, 4, and 8) will be more difficult than a simple experiment in which the set size effect is measured. Ideally, this will involve running an initial experiment to understand the effect sizes due to tDCS, and using those data to feed a power analysis (Reinhart \& Woodman, 2014). In addition, remember that TMS induces changes in neural activity that are a order of magnitude greater than those that result from tDCS. This means that tDCS effect sizes are likely to be significantly smaller than those resulting from other kinds of noninvasive brain stimulation.

Another important consideration is what is done while the stimulation is delivered. As it appears that synaptic changes during stimulation delivery may underlie the after-effects that last for many minutes or even hours after the stimulator is turned off, what you do during stimulation can be important. For example, having participants perform a task of interest during stimulation may have a particularly strong effect in the period after stimulation. However, we typically have our participants just sit quietly during the short stimulation periods and there appears to be ample opportunity to measure interesting changes in the brain after the stimulation has been applied.

The beauty of tDCS for those who also use methods for measuring brain activity is the long-lasting nature of the effects. Because $20 \mathrm{~min}$ of stimulation can offer up to $5 \mathrm{~h}$ of changed brain activity, there is ample time to collect data in the altered state using any electrophysiological or neuroimaging method. After stimulation is complete we take off the stimulating electrodes and either start our behavioral task of interest, or plug the electrode cap into our amplifier and begin recording the EEG and the event-related potentials (ERPs). Similarly, the subject can then be moved into the magnet and MRI scanning can begin.

\section{Combining tDCS with other neuroscientific methods}

The final strength of tDCS that we want to emphasize is that it is easily combined with other methods used to measure neural activity. This is because the effects of tDCS last for several hours, allowing sufficient signal averaging of the changed brain. We will briefly describe a couple of examples in which tDCS was combined with functional MRI and recordings of participants' EEG and the averaged ERPs.

The downside of the long-lasting effects of tDCS is that this type of stimulation results in essentially static changes in the brain. These are slowly evolving effects, and a skeptic might argue that $\mathrm{tDCS}$ provides essentially no temporal resolution. However, when it comes to combining tDCS with fMRI, this can be made to be an advantage. For example, with fMRI it is possible to measure far field effects across the entire brain. When this has been done, researchers have shown that tDCS stimulation can result in changes across a large brain-wide network. Chib, Yun, Takahashi, and Shimojo (2013) provide a nice example of this combination of methods. They showed that stimulation of prefrontal cortex (i.e., anode at Fp1 and cathode at F3) resulted in signal change in the ventral medial cortex of participants viewing face stimuli. That is, this experiment showed that stimulation of relatively remote brain areas can result in far field activations that are not in the current path. This is a natural combination of methods because fMRI excels in measuring whole brain activity to understand the potentially board networks influenced by the tDCS that is long lasting enough to perform the necessary scans.

We might expect tDCS to have poor temporal resolution due to the sluggish nature of this causal manipulation. However, there is evidence from EEG and ERP studies that tDCS can have surprisingly specific effects at certain points in time during the flow of information processing (Reinhart \& Woodman, 2015b). For example, several recent studies showed that tDCS at different locations on the head changed one specific ERP component (lasting approximately $100 \mathrm{~ms}$ ) 
while leaving temporally adjacent components unchanged. One such study showed that tDCS stimulation applied to parietal cortex changed the N1 component elicited by visual stimuli, but not $\mathrm{P} 1$ or N2pc measured just tens of milliseconds on either side of the N1 (Reinhart \& Woodman, 2015b). Another study showed that medial-frontal stimulation changed the error-related negativity (ERN) measured in the first $150 \mathrm{~ms}$ following a response, but no other components during the entire flow of information processing in a visual discrimination task (Reinhart \& Woodman, 2014). This means that the temporal precision of tDCS may be better than we think, changing activity during just one $100-150 \mathrm{~ms}$ period and not any other periods of processing during a trial lasing $1 \mathrm{~s}$ or more. Thus, combining the slow after effects of tDCS with a high temporal resolution technique like electrophysiology can demonstrate that tDCS can have effects with high temporal specificity.

\section{The controversy}

Several cases have been made in the literature and the press arguing that tDCS is not an effective method for manipulating brain activity (Horvath et al., 2015b, a). We completely understand the skepticism with which scientists should view new methods, particularly those that seem to offer causal control over brain activity with low cost and minimal side effects. However, when we dug into the meta-analyses that claimed that tDCS was ineffective (Horvath et al., 2015a,b), we were surprised to see that our own papers were cited as reporting null results. In addition to incorrect citations, others have also noted that these meta-analyses made a number of methodological and conceptual errors that largely invalidate the conclusions that were drawn (Antal et al., 2015; Price \& Hamilton, 2015). It is clearly not consistent with the state of the literature to reject tDCS as a potential tool with which to study the brain.

We approached tDCS from the perspective that it seemed too good to be true. However, the invasive work with animal models clearly shows that it can be effective in changing brain functions (Bindman et al., 1962; Bindman et al., 1964; Rahman et al., 2013). Even with this evidence in hand, the idea that increasing the excitability of large pools of neurons can improve information processing seems unlikely. For example, why is it that increasing the excitability across all neurons does not just increase the noise along with the signal? These questions are active topics of study in our laboratories and those of many others. We are confident that we will make progress understanding the biophysical mechanisms underlying tDCS in the near future.

One view is that tDCS has too often been used to try to enhance cognitive processing and treat disorders, instead of work being focused on trying to understand the nature of the stimulation and how it is changing the brain (Parkin et al., 2014). We agree with this view. However, the critical experiments are probably not possible to conduct with healthy human participants. Instead, understanding the nature of brain stimulation requires recording from brains at microscopic and mesoscopic scales so that the mechanisms that enable the brain to function differently can be understood. Much of the critical work using slice preparations and recording from animals will be needed to understand the biophysical mechanisms that make tDCS a tool that can manipulate activity in the human brain.

A realistic concern that has generated controversy among researchers using brain stimulation is the consumer use of tDCS (Wurzman et al., 2016). Because the technology is not expensive and devices can be constructed with basic components, there has been a rise of do-it-yourself tDCS. Several of the aspects of tDCS that we covered here should make clear the dangers of an uninformed approach to trying to improve one's own cognitive processing. It would be fairly easy to reverse two electrodes and impair cognitive functions, even if a consumer were trying to guide their own use of tDCS using the scientific literature. As we discussed, the effects can last for hours after the administration of stimulation meaning that impaired cognition can take a long time to wear off. Even if no mistakes are made, there can still be unintended consequences. Stimulation can have distant effects on unintended structures (Chib et al., 2013), meaning that someone could stimulate their brain meaning to improve their ability to focus attention on a task, but change their reward sensitivity making them more prone to risky choices. Finally, as we discussed in the section on the biophysical substrates of tDCS, it is still not known exactly how the brain is changing in response to the stimulation. It would be wise for the public to wait until the potential costs of tDCS have been thorough investigated before starting to use it to fine tune the human brain at home.

The tDCS method is far from a panacea. There appear to be cognitive functions that involve neural machinery that are not sufficiently changed by tDCS so as to result in differences in performance. In addition, we have reviewed a number of pitfalls that are easy to fall into when conducting tDCS experiments. We hope that new users will find our suggestions useful and that future work will continue to refine and improve upon the methods currently in use so as to sharpen tDCS as a tool to study cognitive processing.

Acknowledgments The authors declare that they had no conflicts of interest with respect to their authorship or the publication of this article. This work was supported by grants from the National Institutes of Health (R01-EY019882, R01-EY025272, P30-EY08126, F31-MH102042, and T32-EY007135). 


\section{Appendix}

Transcranial direct-current stimulation safety questionnaire

Subject ID:

Date:

Directions

Please circle the appropriate answers to the following questions regarding your experience in this research study, adding information if necessary. Your responses will be kept in the strictest of confidence.

1. Did you experience any feelings of a headache?

Yes No

If so, please rate the severity.

$\begin{array}{llll}\text { Very Mild } & \text { Mild } & \text { Moderate } & \text { Severe }\end{array}$

2. Did you experience any difficulty concentrating? Yes No

Is so, please rate the severity

$\begin{array}{llll}\text { Very Mild } & \text { Mild } & \text { Moderate } & \text { Severe }\end{array}$

3. Did you experience any change in mood?

Yes No

Is so, please rate the severity

$\begin{array}{llll}\text { Very Mild } & \text { Mild } & \text { Moderate } & \text { Severe }\end{array}$

4. Did you experience any change in vision? Yes No

Is so, please rate the severity

Very Mild Mild Moderate Severe Extremely

5. Did you experience any fatigue? $\quad$ Yes No

Is so, please rate the severity

Very Mild Mild Moderate Severe Extremely

6. Did you experience any sensations like pain, tingling, itching, or burning under the electrodes during or after stimulation?

Yes No

Is so, please rate the severity

$\begin{array}{llll}\text { Very Mild } & \text { Mild } & \text { Moderate } & \text { Severe }\end{array}$

\section{Visual Analog Scales (VAS)}

1. Please rate your level of discomfort $(1=$ no discomfort, $10=$ extreme discomfort $)$ ?

$\begin{array}{llllllllll}1 & 2 & 3 & 4 & 5 & 6 & 7 & 8 & 9 & 10\end{array}$

2. Please rate your level of attention and fatigue ( $1=$ least attentive/most fatigue, $10=$ most attentive/least fatigue

$\begin{array}{llllllllll}1 & 2 & 3 & 4 & 5 & 6 & 7 & 8 & 9 & 10\end{array}$




\section{References}

Akhtari, M., Bryant, H. C., Marnelak, A. N., Flynn, E. R., Heller, L., Shih, J. J., ... Sutherling, W. W. (2002). Conductivities of threelayer live human skull. Brain Topography, 14, 151-167.

Alonzo, A., Brassil, J., Taylor, J. L., Martin, D., \& Loo, C. K. (2012). Daily transcranial direct current stimulation (tDCS) leads to greater increases in cortical excitability than second daily transcranial direct current stimulation. Brain Stimulation, 5, 208-213.

Ambrus, G. G., Antal, A., \& Paulus, W. (2011). Comparing cutaneous perception induced by electrical stimulation using rectangular and round shaped electrodes. Clinical Neurophysiology, 122, 803-807.

Antal, A., \& Herrmann, C. S. (2016). Transcranial alternating current and random noise stimulation: Possible mechanisms. Neural Plasticity, 2016, 3616807

Antal, A., Keeser, D., Priori, A., Padberg, F., \& Nitsche, M. A. (2015). Conceptual and procedural shortcomings of the systematic review "Evidence that transcranial direct current stimulation (tDCS) generates little-to-no reliable neurophysiological effect byond MEP amplitdue modulation in healthy human subjects: A systematic review" by Horvath and co-workers. Brain Stimulation, 8, 846-849.

Antal, A., Nitsche, M. A., \& Paulus, W. (2001). External modulation of visual perception in humans. Neuroreport, 16, 3553-3555.

Barbieri, M., Negrini, M., Nitsche, M. A., \& Rivolta, D. (2016). AnodaltDCS over the human right occipital cortex enhances the perception and memory of both faces and objects. Neuropsychologia, 81, 238244.

Baumann, S. B., Wozny, D. R., Kelly, S. K., \& Meno, F. M. (1997). The electrical conductivity of human cerebrospinal fluid at body temperature. IEEE Translations on Biomedical Engineering, 44, 220-223.

Berryhill, M. E., Peterson, D. J., Jones, K. T., \& Stephens, J. A. (2014). Hits and misses: Leveraging tDCS to advance cognitive research. Frontiers in Psychology, 5, 800.

Berryhill, M., Wencil, E., Branch Coslett, H., \& Olson, I. (2010). A selective working memory impairment after transcranial direct current stimulation to the right parietal lobe. Neuroscience Letters, 479, 312-316.

Bikson, M., Grossman, P., Thomas, C., Zannou, A. L., Jiang, J., Adnan, T., ... Woods, A. J. (2016). Safety of transcranial direct current stimulation: Evidence based update 2016. Brain Stimulation, 9, 641-661.

Bikson, M., Rahman, A., \& Datta, A. (2012). Computational models of transcranial direct current stimulation. Clinical EEG and Neuroscience, 43, 176-183.

Bindman, L. J., Lippold, O. C. J., \& Redfearn, J. W. T. (1962). Long lasting changes in the level of the electrical activity of the cerebral cortex produced by polarizing currents. Nature, 196, 584-585.

Bindman, L. J., Lippold, O. C. J., \& Redfearn, J. W. T. (1964). The action of brief polarizing currents on the cerebral cortex of the rat (1) during current flow and (2) in the production of long-lasting after-effects. Journal of Physiology, 172, 369-382.

Boot, W. R., Simons, D. J., Stothart, C., \& Stutts, C. (2013). The pervasive problem with placebos in psychology: Why active control groups are not sufficient to rule out placebo effects. Perspectives on Psychological Science : A Journal of the Association for Psychological Science, 8, 445-454.

Burger, H. C., \& van Milaan, J. B. (1943). Measurements of the specific resistance of the human body to direct current. Acta Medica Scandinavica, 114, 584-607.

Chib, V. S., Yun, K., Takahashi, H., \& Shimojo, S. (2013). Noninvasive remote activation of the ventral midbrain by transcranial direct current stimulation of prefrontal cortex. Translational Psychiatry, 3, e268.

Clark, V. P., Coffman, B. A., Mayer, A. R., Weisend, M. P., Lane, T. D. R., Calhoun, V. D., ... Wasserman, E. M. (2012). TDCS guided using fMRI significantly accelerates learning to identify concealed objects. NeuroImage, 59, 117-128.
Clark, V. P., Fan, S., \& Hillyard, S. A. (1995). Identification of early visually evoked potential generators by retinotopic and topographic analyses. Human Brain Mapping, 2, 170-187.

Corbetta, M., \& Shulman, G. L. (2002). Control of goal-directed and stimulus-driven attention in the brain. Nature Reviews Neuroscience, 3, 201-215.

Cosman, J. D., Atreya, P. V., \& Woodman, G. F. (2015). Transient reduction of visual distraction following electrical stimulation of the prefrontal cortex. Cognition, 145, 73-76.

Creutzfeldt, O. D., Fromm, G. H., \& Kapp, H. (1962). Influence of transcortical d-c currents on cortical neuronal activity. Experimental Neurology, 5, 436-452.

Dannhauer, M., Lanfer, B., Wolters, C. H., \& Knösche, T. R. (2011). Modeling of the human skull in EEG source analysis. Human Brain Mapping, 32, 1383-1399.

DaSilva AF, Volz MS, Bikson M, Fregni F (2011) Electrode positioning and montage in transcranial direct current stimulation. Journal of Visualized Experiments, 51, 2744.

De Lucia, M., Parker, G. J., Embleton, K., Newton, J. M., \& Walsh, V. (2007). Diffusion tensor MRI-based estimation of the influence of brain tissue anisotropy on the effects of transcranial magnetic stimulation. NeuroImage, 36, 1159-1170.

Dmochowski, J. P., Datta, A., Bikson, M., Su, Y., \& Parra, L. C. (2011). Optimized multi-electrode stimulation increase focality and intensity at target. Journal of Neural Engineering, 8, 046011.

Dumel, G., Bourassa, M. E., Desjardins, M., Voarino, N., CharleboisPlante, C., Doyon, J., \& De Beaumont, L. (2016). Multisession anodal tDCS protocol improves motor system function in an aging population. Neural Plasticity, 2016, 5961362.

Dundas, J. E., Thickbroom, G. W., \& Mastaglia, F. L. (2007a). Perception of comfort during transcranial DC stimulation: Effect of $\mathrm{NaCl}$ solution concentration applied to sponge electrodes. Clinical Neurophysiology, 118, 1166-1170.

Dundas, J. E., Thickbroom, G. W., \& Mastaglia, F. L. (2007b). Perception of comfort during transcranial direct current stimulation: Effect of $\mathrm{NaCl}$ solution concentration applied to sponge electrodes. Clinical Neurophysiology, 118, 1166-1170.

Filmer, H. L., Dux, P. E., \& Mattingley, J. B. (2014). Applications of transcranial direct current stimulation for undestanding brain function. Trends in Neurosciences, 37, 742-753.

Fregni, F., Boggio, P., Lima, M., Ferreira, M., Wagner, T., Rigonatti, S., ... Pascual-Leone, A. (2006a). A sham-controlled, phase II trial of transcranial direct current stimulation for the treatment of central pain in traumatic spinal cord injury. Pain, 122, 197209.

Fregni, F., Boggio, P. S., Nitsche, M. A., Rigonatti, S. P., \& PascualLeone, A. (2006b). Cognitive effects of repeated sessions of transcranial direct current stiulation in patients with depression. Depression and Anxiety, 23, 482-484.

Fresnoza, S., Paulus, W., Nitsche, M. A., \& Kuo, M. F. (2014a). Nonlinear dose-dependent impact of D1 receptor activation on motor cortex plasticity in humans. Journal of Neuroscience, 34, 2744 2753.

Fresnoza, S., Stiksrud, E., Klinker, F., Liebetanz, D., Paulus, W., Kuo, M. F., \& Nitsche, M. A. (2014b). Dosage-dependent effect of dopamine D2 receptor activation on motor cortex plasticity in humans. Journal of Neuroscience, 34, 10701-10709.

Galvez, V., Alonzo, A., Martin, D., \& Loo, C. K. (2013). Transcranial direct current stimulation treatment protocols: Should stimulus intensity be constant or incremental over multiple sessions? The International Journal of Neuropsychopharmacology / Official Scientific Journal of the Collegium Internationale Neuropsychopharmacologicum (CINP), 16, 13-21.

Gandiga, P., Hummel, F., \& Cohen, L. (2006). Transcranial DC stimulation (tDCS): A tool for double-blind sham-controlled clinical studies in brain stimulation. Clinical Neurophysiology, 117, 845-850. 
Gartside, I. B. (1968). Mechanisms of sustained increases of firing rate of neurones in the rat cerebral cortex after polarization: Role of protein synthesis. Nature, 220, 383-384.

Gick, M., \& Holyoak, K. J. (1980). Analogical problem solving. Cognitive Psychology, 12, 306-355.

Haueisen, J., Ramon, C., Eiselt, M., Brauer, H., \& Nowak, H. (1997). Influence of tissue resistivities on neuromagnetic fields and electric potentials studied with a finite element model of the head. IEEE Translations on Biomedical Engineering, 44, 727-735.

Horvath, J. C., Forte, J. D., \& Carter, O. (2015a). Evidence that transcranial direct current stimulation (tDCS) generates little-to-no reliable neurophysiologic effect beyond MEP amplitude modulation in healthy human subjects: A systematic review. Neuropsychologia, 66, 213-236.

Horvath, J. C., Forte, J. D., \& Carter, O. (2015b). Quantitative review finds no evidence of cognitive effects in healthy populations from single-session transcranial direct current stimulation (tDCS). Brain Stimulation, 8, 535-550.

Hsu, T., Tseng, L., Yu, J., Kuo, W., Hung, D., Tzeng, O., ... Juan, C. (2011). Modulating inhibitory control with direct current stimulation of the superior medial frontal cortex. NeuroImage, 56, 2249-2257.

Iyer, M. B., Mattu, U., Grafman, J., Lomarev, M., Sato, S., \& Wassermann, E. M. (2005). Safety and cognitive effect of frontal DC brain polarization in healthy individuals. Neurology, 64, 872875.

Jasper, H. H. (1958). The ten-twenty electrode system of the International federation. Electroencephalography \& Clinical Neurophysiology, 10, 371-375.

Kadosh, R. C., Soskic, S., Iuculano, T., Kanai, R., \& Walsh, V. (2010). Modulating neuronal activity produces specific and long-lasing changes in numerical competence. Current Biology, 20, 2016-2020.

Keshvari, F., Pouretemad, H.-R., \& Ekhitari, H. (2013). The polaritydependent effects of bilateral brain stimulation on working memory. Basic and Clinical Neuroscience, 4, 224-231.

Kuo, M. F., \& Nitsche, M. A. (2012). Effects of transcranial electrical stimulation on cognition. Clinical EEG and Neuroscience, 43, 192199

Liebetanz, D., Koch, R., Mayenfels, S., Konig, F., Paulus, W., \& Nitsche, M. A. (2009). Safety limits of cathodal transcranial direct current stimulation in rats. Clinical Neurophysiology, 120, 1161-1167.

Liebetanz, D., Nitsche, M. A., Tergau, F., \& Paulus, W. (2002). Pharmacological approach to the mechanisms of DC-stimulation induced after-effects of human motor cortex excitability. Brain, $125,2238-2247$.

Liu, A., Bryant, A., Jefferson, A., Friedman, D., Minhas, P., Barnard, S., ... Schachter, S. (2016). Exploring the efficacy of a 5-day course of transcranial direct current stimulation (TDCS) on depression and memory function in patients with well-controlled temporal lobe epilepsy. Epilepsy and Behavior, 55, 11-20.

Luck, S. J. (2005). An introduction to the event-related potential technique. Cambridge, MA: MIT Press.

Markov, N. T., Vezoli, J., Chameau, P., Falchier, A., Quilodran, R., Huissoud, C., ... Kennedy, H. (2014). Anatomy of hierarchy: Feedforward and feedback pathways in macaque visual cortex. Journal of Comparative Neurology, 522, 225-259.

Medeiros, L. F., de Souza, I. C., Vidor, L. P., de Souza, A., Deitos, A., Volz, M. S., ... Torres, I. L. (2012). Neurobiological effects of transcranial direct current stimulation: A review. Frontiers in Psychiatry, 3,110 .

Neggers, S. F. W., Langerak, R., Mandl, R., Schutter, D., Lemmens, P. J. J., Postma, A., \& Ramsey, N. F. (2004). A stereotactic method for image guided transcranial magnetic stimulation validated with $\mathrm{AMRI}$ and motor evoked potentials. NeuroImage, 21, 1805-1817.

Nitsche, M. A., Cohen, L. G., Wassermann, E. M., Priori, A., Lang, N., Antal, A., ... Pascual-Leone, A. (2008). Transcranial direct current stimulation: State of the art 2008. Brain Stimulation, 1, 206-223.
Nitsche, M. A., Fricke, K., Henschke, U., Schlitterlau, A., Liebetanz, D., Lang, N., ... Paulus, W. (2003b). Pharmacological modulation of cortical excitability shifts induced by transcranial direct current stimulation in humans. Journal of Physiology, 553, 293-301.

Nitsche, M. A., Grundey, J., Liebetanz, D., Lang, N., Tergau, F., \& Paulus, W. (2004). Catecholaminergic consolidation of motor cortical neuroplasticity in humans. Cerebral Cortex, 14, 1240-1245.

Nitsche, M., Liebetanz, D., Antal, A., Lang, N., Tergau, F., \& Paulus, W. (2003a). Modulation of cortical excitability by weak direct current stimulation-technical, safety and functional aspects. Supplements to Clinical Neurophysiology, 56, 255-276.

Nitsche, M. A., Liebetanz, D., Lang, N., Antal, A., Tergau, F., \& Paulus, W. (2003c). Safety criteria for transcranial direct current stimulation (tDCS) in humans. Clinical Neurophysiology, 114, 2220-2222.

Nitsche, M., \& Paulus, W. (2001). Sustained excitability elevations induced by transcranial DC motor cortex stimulation in humans. Neurology, 57, 1899-1901.

Nozari, N., Woodward, K., \& Thompson-Shill, S. L. (2014). Consequences of cathodal stimulation for behavior: When does it help and when does it hurt performance. Public LIbrary of Sciences Biology, 9, e84338.

Nunez, P. L., \& Srinivasan, R. (2006). Electric fields of the brain: The neurophysics of EEG. Oxford: Oxford University Press, Inc.

Oostendorp, T. F., Delbeke, J., \& Stegeman, D. F. (2000). The conductivity of the human skull: Results of in vivo and in vitro measurements. IEEE Translations on Biomedical Engineering, 47, 14871492.

Oostenveld, R., Fries, P., Maris, E., \& Schoffelen, J. M. (2011). FieldTrip: Open source software for advanced analysis of MEG, EEG, and invasive electrophysiological data. Computational Intelligence and Neuroscience, 2011, 1-9.

Paneri, B., Adair, D., Thomas, C., Khadka, N., Patel, V., Tyler, W. J., ... Bikson, M. (2016). Tolerability of repeated application of transcranial electrical stimulation with limited outputs to healthy subjects. Brain Stimulation, 9, 740-754.

Parkin, B. L., Ekhitiari, H., \& Walsh, V. (2014). Non-invasiv human brain stimulation in cognitive neuroscience: A primer. Neuron, 87, 932 946.

Paulus, W. (2011). Transcranial electrical stimulation (tES - tDCS; tRNS, tACS) methods. Neuropsychological Rehabilitation, 21, 602-617.

Poreisz, C., Boros, K., Antal, A., \& Paulus, W. (2007). Safety aspects of transcranial direct current stimulation concerning healthy subjects and patients. Brain Research Bulletin, 72, 208-214.

Price, A. R., \& Hamilton, R. H. (2015). A re-evaluation of the congitive effects from single-session transcranial direct current stimulation. Brain Stimulation, 8, 663-665.

Purpura, D. P., \& McMurtry, J. G. (1965). Intracellular activities and evoked potential changes during polarization of motor cortex. Journal of Neurophysiology, 28, 166-185.

Radman, T., Ramos, R. L., Brumberg, J. C., \& Bikson, M. (2009). Role of cortical cell type and morphology in subthreshold and suprathreshold uniform electric field stimulation in vitro. Brain Stimulation, 2, 215-228.

Rahman, A., Reato, D., Arlotti, M., Gasca, F., Datta, A., Parra, L. C., \& Bikson, M. (2013). Cellular effects of acute direct current stimulation: Somatic and synaptic terminal effects. Journal of Physiology, 591, 2563-2578.

Raimundo, R. J., Uribe, C. E., \& Brasil-Neto, J. P. (2012). Lack of clinically detectable acute changes on autonomic or thermoregulatory functions in healthy subjects after transcranial direct current stimulation (tDCS). Brain Stimulation, 5, 196-200.

Reato, D., Rahman, A., Bikson, M., \& Parra, L. C. (2013). Effects of weak transcranial alternating current stimulation on brain activity-a review of known mechanisms from animal studies. Frontiers in Human Neuroscience, 7, 687. 
Reinhart, R. M., \& Woodman, G. F. (2014). Causal control of medialfrontal cortex governs electrophysiological and behavioral indices of performance monitoring and learning. Journal of Neuroscience, 34 , 4214-4227.

Reinhart, R. M., \& Woodman, G. F. (2015a). Enhancing long-term memory with stimulation tunes visual attention in one trial. Proceedings of the National Academy of Sciences of the United States of America, 112, 625-630.

Reinhart, R. M., \& Woodman, G. F. (2015b). The surprising temporal specificity of direct-current stimulation. Trends in Neurosciences, $38,459-461$.

Reinhart, R. M., Xiao, W., McClenahan, L. J., \& Woodman, G. F. (2016). Electrical stimulation of visual cortex can immediately improve spatial vision. Current Biology, 26, 1867-1872.

Reinhart, R. M., Zhu, J., Park, S., \& Woodman, G. F. (2015). Synchronizing theta oscillations with direct-current stimulation strengthens adaptive control in the human brain. Proceedings of the National Academy of Sciences of the United States of America, 112, 9448-9453.

Reis, J., \& Fritsch, B. (2011). Modulation of motor performance and motor learning by transcranial direct current stimulation. Current Opinion in Neurobiology, 24, 590-596.

Rueger, M. A., Keuters, M. H., Walberer, M., Braun, R., Klein, R., Sparing, R., ... Schroeter, M. (2012). Multi-session transcranial direct current stimulation (tDCS) elicits inflammatory and regenerative processes in the rat brain. PloS One, 7, e43776.

Rush, S., \& Driscoll, D. A. (1968). Current distribution in the brain from surface electrodes. Anesthesia and Analgesia, 47, 717-723.

Rushmore, R. J., DeSimone, C., \& Valero-Cabré, A. (2013). Multiple sessions of transcranial direct current stimulation to the intact hemisphere improves visual function after unilateral ablation of visual cortex. European Journal of Neuroscience, 38, 3799-3807.

Sadleir, R. J., Vannorsdall, T. D., Schretlen, D. J., \& Gordon, B. (2010). Transcranial direct current stimulation (tDCS) in a realistic head model. NeuroImage, 51, 1310-1318.

Santarnecchi, E., Brem, A.-K., Levenbaum, E., Thompson, T., Kadosh, R. C., \& Pascual-Leone, A. (2015). Enhancing cognition using transcranial electrical stimulation. Current Opinion in Behavioral Sciences, 4, 171-178.

Schall, J. D. (1997). Visuomotor areas of the frontal lobe. In K. S. Rockland, J. H. Kaas, \& A. Peters (Eds.), Cerebral cortex, vol. 4 (pp. 527-638). New York: Plenum.
Serences, J. T., \& Yantis, S. (2006). Selective visual attention and perceptual coherence. Trends in Cognitive Sciences, 10, 38-45.

Shekhawat, G. S., Sundram, F., Bikson, M., Truong, D., De Ridder, D., Stinear, C. M., ... Searchfield, G. D. (2015). Intensity, duration, and location of high-definition transcranial direct current stimulation for tinnitus relief. Neurorehabilitation and Neural Repair, 30, 349-359.

Stagg, C. J., \& Nitsche, M. A. (2011). Physiological basis of transcranial direct current stimulation. The Neuroscientist, 17, 37-53.

Sun, Y., Lipton, J. O., Boyle, L. M., Madsen, J. R., Goldenberg, M. C., Pascual-Leone, A., ... Rotenberg, A. (2016). Direct current stimulation induces mGluR5-dependent neocortical plasticity. Annals of Neurology, 80, 233-246.

Syková, E., \& Nicholson, C. (2008). Diffusion in brain extracellular space. Physiological Reviews, 88, 1277-1340.

Tseng, P., Hsu, T.-Y., Chang, C.-F., Tzeng, O., Hung, D., Muggleton, N., ... Juan, C.-H. (2012). Unleashing potential: Transcranial direct current stimulation over the right posterior parietal cortex improves change detection in low-performing individuals. Journal of Neuroscience, 32, 10554-10561.

Tuch, D. S., Wedeen, V. J., Dale, A. M., George, J. S., \& Belliveau, J. W. (2001). Conductivity tensor mapping of the human brain using diffusion tensor MRI. Proceedings of the National Academy of Sciences of the USA, 98, 11697-11701.

Utz, K., Dimova, V., Oppenländer, K., \& Kerkhoff, G. (2010). Electrified minds: transcranial direct current stimulation (tDCS) and galvanic vestibular stimulation (GVS) as methods of non-invasive brain stimulation in neuropsychology: A review of current data and future implications. Neuropsychologia, 48, 2789-2810.

Van Essen, D. C., \& Dierker, D. (2007). On navigating the human cortex. NeuroImage, 37, 1050-1054.

Wagner, T., Fregni, F., Fecteau, S., Grodzinsky, A., Zahn, M., \& Pascual-Leone, A. (2007). Transcranial direct current stimulation: A computer-based human model study. NeuroImage, 35, 11131124.

Woodman, G. F. (2010). A brief introduction to the use of event-related potentials (ERPs) in studies of perception and attention. Attention, Perception \& Psychophysics, 72, 2031-2046.

Wurzman, R., Hamilton, R. H., Pascual-Leone, A., \& Fox, M. D. (2016). An open letter concerning do-it-yourself users of transcranial direct current stimulation. Annals of Neurology, 80, 1-4. 